\title{
Correlation Inequalities and the Thermodynamic Limit for Classical and Quantum Continuous Systems ${ }^{\star}$
}

\author{
Jürg Fröhlich ${ }^{\star \star}$ \\ Department of Mathematics, Princeton University, Princeton, NJ 08540, USA \\ Yong Moon Park \\ Department of Mathematics, Yonsei University, Seoul, Korea
}

\begin{abstract}
We use Ginibre's general formulation of Griffiths' inequalities to derive new correlation inequalities for two-component classical and quantum mechanical systems of distinguishable particles interacting via two body potentials of positive type. As a consequence we obtain existence of the thermodynamic limit of the thermodynamic and correlation functions in the grand canonical ensemble at arbitrary temperatures and chemical potentials. For a large class of systems we show that the limiting correlation functions are clustering. (In a subsequent article these results are extended to the correlation functions of two-component quantum mechanical gases with Bose-Einstein statistics). Finally, a general construction of the thermodynamic limit of the pressure for gases which are not $\mathrm{H}$-stable, above collapse temperature, is presented.
\end{abstract}

\section{Systems of Particles Interacting via Two Body Potentials of Positive Type}

In this paper we study classical and quantum continuous systems in thermal equilibrium. These systems consist of particles the interactions among which are described by two body potentials of positive type. We are interested in proving the existence of the thermodynamic limit of the pressure and the Gibbs equilibrium states in the grand canonical ensemble. We also want to discuss certain properties of the equilibrium states in the thermodynamic limit, such as clustering. Two classes of systems are considered:

(C) Classical particles, and

(QM) Quantum mechanical, distinguishable particles, ("Boltzmann statistics").

The particles have internal degrees of freedom which we call (generalized) charges. They are labelled by the vectors $q$ of some measurable vector space $Q$.

* Research supported in part by the U.S. National Science Foundation under grant MPS 75-11864

$\star \star$ A Sloan Foundation Fellow 
When the dimension of space is 3 we may think of a particle as being some ion with a net charge $\varepsilon \in \mathbb{R}$ (or $\varepsilon \in \mathbb{Z}$ ), a dipole moment $\underline{\varepsilon} \in \mathbb{R}^{3}$ (or $\underline{\varepsilon} \in S^{2}$, the unit sphere in $\mathbb{R}^{3}$ ) and possibly higher multipole moments, so that typically

$$
Q=\mathbb{R} \oplus \mathbb{R}^{3} \oplus \mathbb{R}^{5} \oplus \ldots
$$

In this situation $q=(\varepsilon, \underline{\varepsilon}, \underline{\varepsilon}, \ldots)$. The potential between a particle with generalized charge $q$ at position $x \in \mathbb{R}^{v}$ and one with generalized charge $q^{\prime}$ at $x^{\prime} \in \mathbb{R}^{v}$ is given by a function $V\left(q, x ; q^{\prime}, x^{\prime}\right)$ on $\left(Q \times \mathbb{R}^{v}\right)^{\times 2}$ which we require to be of positive type, i.e., given an arbitrary sequence of complex numbers $\left\{c_{j}\right\}_{j=1}^{N}$ and a sequence of points in $Q \times \mathbb{R}^{v},\left\{\left(q_{j}, x_{j}\right)\right\}_{j=1}^{N}$,

$$
\sum_{i, j=1}^{N} \bar{c}_{i} c_{j} V\left(q_{i}, x_{i} ; q_{j}, x_{j}\right) \geq 0 \text {. }
$$

We set

$$
\left.\begin{array}{l}
(q)_{N}=\left(q_{1}, \ldots, q_{N}\right),(x)_{N}=\left(x_{1}, \ldots, x_{N}\right) \\
d \lambda(q)_{N}=\prod_{j=1}^{N} d \lambda\left(q_{j}\right), d(x)_{N}=\prod_{j=1}^{N} d^{v} x_{j}
\end{array}\right\} .
$$

Here $\lambda$ is some finite, positive measure on $Q$ with the property that

$$
d \lambda(q)=d \lambda(-q),
$$

and $V$ is required to satisfy

$$
\left.V\left(q, x ; q^{\prime}, x^{\prime}\right)=-V\left(-q, x ; q^{\prime}, x^{\prime}\right)=-V\left(q, x ;-q^{\prime}, x^{\prime}\right)\right\} .
$$

Condition (1.4) is a neutrality - or charge symmetry condition.

The potential for $N$ particles at positions $(x)_{N}$ and with charges $(q)_{N}$ is given by

$$
U\left((q)_{N},(x)_{N}\right)=\sum_{1 \leq i<j \leq N} V\left(q_{i}, x_{i} ; q_{j}, x_{j}\right) .
$$

Examples. (A) $Q=\mathbb{R}, q=$ charge, $d \lambda(q)=\frac{1}{2}\{\delta(q-1)+\delta(q+1)\} d q$

$$
V\left(q, x ; q^{\prime}, x^{\prime}\right)=q \cdot q^{\prime}(2 \pi)^{-v / 2} \int e^{i k\left(x-x^{\prime}\right)} \hat{V}(k) d^{v} k,
$$

with $0 \leq \hat{V}(k) \in L^{1}\left(\mathbb{R}^{v}\right)$.

(B) $Q=\mathbb{R}^{v}, q=\underline{\varepsilon}=$ dipole moment, $d \lambda(\underline{\varepsilon})=\delta(|\underline{\varepsilon}|-1) d^{v} \varepsilon$,

$V\left(\underline{\varepsilon}, x ; \underline{\varepsilon}^{\prime}, x^{\prime}\right)=(2 \pi)^{-v / 2} \int e^{i k\left(x-x^{\prime}\right)}(\underline{\varepsilon} \cdot k)\left(\underline{\varepsilon}^{\prime} \cdot k\right) \hat{W}(k) d^{v} k$, with $0 \leq|k|^{2} \hat{W}(k) \in L^{1}\left(\mathbb{R}^{y}\right)$.

This is a dipole potential.

Clearly there may also be dipole-monopole potentials, (when $\hat{V}=\hat{W}$ ), and we can accommodate potentials with infrared singularities such as the two dimensional Coulomb potential [6], so that e.g. $\hat{V}(k) \geq 0$, for $k \neq 0$ only.

The grand canonical partition function for the classical system in a compact 
region $\Lambda \subset \mathbb{R}^{v}$ is defined by

$$
\Xi_{\Lambda}(\beta, z)=\sum_{N=0}^{\infty} \frac{z^{N}}{N !} \int_{Q^{N}} d \lambda(q)_{N} \int_{\Lambda^{N}} d(x)_{N} e^{-\beta U\left((q)_{N},(x)_{N}\right)}
$$

with $0 \leq z<\infty, 0 \leq \beta<\infty$, and the term corresponding to $N=0$ is defined to be $=1$. The pressure of the system in the region $\Lambda$ is given by

$$
p_{\Lambda}(\beta, z)=\frac{1}{|\Lambda|} \log \Xi_{\Lambda}(\beta, z)
$$

and the correlation functions are defined by

$$
\begin{aligned}
\rho_{\Lambda}\left(\beta, z ;(q)_{N},(x)_{N}\right)=\Xi_{\Lambda}(\beta, z)^{-1} z^{N}[ & \sum_{M=0}^{\infty} \frac{z^{M}}{M !} \\
& \left.\cdot \int_{Q^{M}} d \lambda\left(q^{\prime}\right)_{M} \int_{\Lambda^{M}} d\left(x^{\prime}\right)_{M} e^{-\beta U\left((q)_{N}\left(q^{\prime}\right)_{M},(x)_{N}\left(x^{\prime}\right)_{M}\right)}\right] .
\end{aligned}
$$

We shall prove

Theorem C. Assume that $\sup \int d \lambda(q) e^{\alpha V(q, x ; q, x)}<\infty$, for all $\alpha>0$.

$$
x \in \mathbb{R}^{v} Q
$$

Then, for arbitrary $\beta \geq 0, z \geq 0$ and an arbitrary sequence of compact regions $\{\Lambda\}$ increasing to $\mathbb{R}^{v}$,

(1) if $V$ is translation invariant $p(\beta, z)=\lim _{\Lambda \uparrow \mathbb{R}^{v}} p_{\Lambda}(\beta, z)$ exists and is independent of the sequence chosen, and $p(\beta, z)$ has the standard thermodynamic properties of the pressure.

(2) $\rho\left(\beta, z ;(q)_{N}(x)_{N}\right)=\lim _{\Lambda^{\uparrow} \mathbb{R}^{v}} \rho_{\Lambda}\left(\beta, z ;(q)_{N},(x)_{N}\right)$

exists, for all $N=0,1,2, \ldots$ It is monotone increasing in $z$ and $\lambda$ and is bounded by

$$
z^{N} e^{(\beta / 2) \sum_{j=1}^{N} V\left(q_{j}, x_{j} ; q_{j}, x_{j}\right)},
$$

for all $z \geq 0$.

Results of the type of Theorem $\mathrm{C}$ have earlier been proven for a large variety of systems under various conditions, [25]. The existence and shape independence of the thermodynamic limit for the pressure has already been demonstrated for a large class of potentials of not too long range; see [25] and Refs. given there; for results concerning the two dimensional Yukawa and Coulomb gas, see [6]. Correlation functions for systems with potentials of not too long range at small $z$ and $\beta$ have also been constructed; see [25] and Refs. given there. For the two dimensional Yukawa gas above collapse temperature these results were proven in $[6,8]$, for the Coulomb gas in [24].

The novel aspect of Theorem $\mathrm{C}$ is that it does not require any restrictions on the range of $V$ or on the values of $z \geq 0$ and $\beta \geq 0$. The thermodynamic limits of pressure and correlation functions claimed to exist in Theorem $\mathrm{C}$ are shape independent and have all the symmetries of the potential $V$. Theorem $\mathrm{C}$ is an existence theorem for systems as little explored as the classical dipole gas. 
To prove Theorem $\mathrm{C}$ we make use of functional integrals as in [6] and apply some generalization of the correlation inequalities of [24]. Correlation inequalities were first applied in [6] to prove the existence of the thermodynamic limit of the correlation functions of the two dimensional Yukawa gas. Some extensions were presented in [8]. But the most useful inequalities were discovered in [24].

In order to extend Theorem $\mathrm{C}$ to the quantum mechanical systems with distinguishable particles we use the conditional Wiener measure as in [12], in addition to functional integrals of the sort applied in the classical case. This enables us to prove the necessary correlation inequalities. We consider $N$ distinguishable particles with charges $q_{1}, \ldots, q_{N}$ and masses $m\left(q_{1}\right), \ldots, m\left(q_{N}\right)$. The Hilbert space for these particles confined to a compact region $\Lambda \subset \mathbb{R}^{v}$ is defined by

$$
\mathscr{H}_{\Lambda}^{N}=\bigotimes_{j=1}^{N} L^{2}\left(\Lambda, d^{v} x_{j}\right)
$$

Let $\Delta_{i}^{\Lambda}$ be the Laplacean in the variables $x_{i}$ with O-Dirichlet data at the boundary $\partial \Lambda$ of $\Lambda$. The Hamiltonian for these particles is given by

$$
H_{\Lambda}^{N}\left((q)_{N}\right)=-\sum_{i=1}^{N} \frac{\Delta_{i}^{A}}{2 m\left(q_{i}\right)}+U\left((q)_{N},(x)_{N}\right)
$$

with $0<\underline{m}=\inf _{q \in Q} m(q) \leq \sup _{q \in Q} m(q)=\bar{m}<\infty$, and $m(q)=m(-q)$.

We also assume that

$$
\sup _{q \in Q} \sup _{x \in \mathbb{R}^{v}} V(q, x ; q, x)<\infty \text {. }
$$

In this case $H_{A}^{N}\left((q)_{N}\right)$ is essentially selfadjoint on a natural dense domain in $\mathscr{H}_{\Lambda}^{N}$, for all $(q)_{N} \in Q^{N}$. Moreover $\exp \left[-\beta H_{\Lambda}^{N}\left((q)_{N}\right)\right]$ is trace class, for bounded, open $\Lambda$ and $\beta>0$.

Let $P_{\Lambda}^{\beta}\left((q)_{N} ;(x)_{N},\left(x^{\prime}\right)_{N}\right)$ denote the kernel of $\exp \left[-\beta H_{\Lambda}^{N}\left((q)_{N}\right)\right]$. For $\beta>0$, it is well defined, positive and continuous in $(x)_{N}$ and $\left(x^{\prime}\right)_{N}$. Thus we may define

$$
\psi_{\Lambda}^{\beta}\left((q)_{N},(x)_{N}\right)=P_{\Lambda}^{\beta}\left((q)_{N} ;(x)_{N},(x)_{N}\right) .
$$

As usual, the grand canonical partition function is defined by

$$
\Xi_{\Lambda}(\beta, z)=\sum_{N=0}^{\infty} \frac{z^{N}}{N !} \int_{Q^{N}} d \lambda(q)_{N} \operatorname{Tr}_{\mathscr{C}_{\Lambda}^{N}}\left(e^{-\beta H_{\Lambda}^{N}\left((q)_{N}\right)}\right),
$$

with the term corresponding to $N=0$ set $=1$. The continuity of $P_{A}^{\beta}\left((q)_{N} ;(x)_{N}\right.$, $\left.\left(x^{\prime}\right)_{N}\right)$ in a neighborhood of $(x)_{N}=\left(x^{\prime}\right)_{N}$ and (1.12) permit us to rewrite $\Xi_{\Lambda}(\beta, z)$ as follows:

$$
\left.\Xi_{\Lambda}(\beta, z)=\sum_{N=0}^{\infty} \frac{z^{N}}{N !} \int_{Q^{N}} d \lambda(q)_{N} \int_{\Lambda^{N}} d(x)_{N} \psi_{\Lambda}^{\beta}(q)_{N},(x)_{N}\right) .
$$

The pressure is still given by (1.7), i.e.

$$
p_{\Lambda}(\beta, z)=\frac{1}{|\Lambda|} \log \Xi_{\Lambda}(\beta, z),
$$


and the (particle-) density correlation functions for the system in the region $\Lambda$ by

$$
\begin{aligned}
\rho_{\Lambda}\left(\beta, z ;(q)_{N},(x)_{N}\right)= & \Xi_{\Lambda}(\beta, z)^{-1} z^{N}\left[\sum_{M=0}^{\infty} \frac{z^{M}}{M !}\right. \\
& \left.\cdot \int_{Q^{M}} d \lambda\left(q^{\prime}\right)_{M} \int_{\Lambda^{M}} d\left(x^{\prime}\right)_{M} \psi_{\Lambda}^{\beta}\left((q)_{N}\left(q^{\prime}\right)_{M},(x)_{N}\left(x^{\prime}\right)_{M}\right)\right] .
\end{aligned}
$$

In Appendix 1 we introduce the correlation - and imaginary time ("temperature ordered") Green's functions (ITGF's) for these quantum mechanical systems and indicate how to construct their thermodynamic limit, using methods developed in Sections 3 and 4.

Next, we briefly recall definition and properties of conditional Wiener measures $[22,12]$. Let

$$
\Omega=\underset{\tau \in[0, \beta]}{\times} \dot{\mathbb{R}}_{\tau}^{v}
$$

where $\mathbb{R}_{\tau}^{v}$ is a copy of the one point compactification of $\mathbb{R}^{v}$. Clearly $\Omega$ is a compact Hausdorff space with $\sigma$-algebra the Borel sets. It serves as a measure space on which the conditional Wiener measure $\operatorname{Pr}_{\Lambda}^{\beta}(q, x, y ; d \omega)$ can be defined as a $\sigma$-additive, finite measure. This is the path space measure for the Wiener process with transition function $\exp \left[t \Delta^{\Lambda} / 2 m(q)\right]$ conditioned on the set of paths in $\Omega$ with $\omega(\tau=0)=x \in \Lambda, \omega(\tau=\beta)=y \in \Lambda$. We set

$$
\operatorname{Pr}_{\Lambda}^{\beta}\left((q)_{N},(x)_{N},\left(x^{\prime}\right)_{N} ; d(\omega)_{N}\right)=\prod_{j=1}^{N} \operatorname{Pr}_{\Lambda}^{\beta}\left(q_{j}, x_{j}, x_{j}^{\prime} ; d \omega_{j}\right) \text {. }
$$

Applying the Feynman-Kac formula one sees that

$$
\begin{aligned}
P_{\Lambda}^{\beta}\left((q)_{N} ;(x)_{N},\left(x^{\prime}\right)_{N}\right) & =\int_{\Omega^{N}} \operatorname{Pr}_{\Lambda}^{\beta}\left((q)_{N},(x)_{N},\left(x^{\prime}\right)_{N} ; d(\omega)_{N}\right) \\
& \cdot e^{-\int_{0}^{\beta} d \tau U\left((q)_{N},(\omega(\tau))_{N}\right) .}
\end{aligned}
$$

Combining (1.15), (1.16) and (1.18) with functional integrals expressing $\exp \left[-\int_{0}^{\beta} d \tau U\left((q)_{N},(\omega(\tau))_{N}\right)\right]$ and an extension of the correlation inequalities of [24] we shall be able to prove

Theorem QM. Theorem $C$ is true for the pressure (see (1.15)) and the density correlation functions (see (1.16)) of the quantum mechanical system with Boltzmann statistics and two body potential $V\left(q, x ; q^{\prime}, x^{\prime}\right)$, except that the upper bound is now given by

$$
\left(z\left(\frac{2 \pi \bar{m}}{\beta}\right)^{v / 2}\right)^{N} \exp \left[\frac{\beta}{2} \sum_{j=1}^{N} \sup _{x} V\left(q_{j}, x ; q_{j}, x\right)\right] .
$$

Remarks. (1) In Appendix 1, Theorem QM is extended to the correlation functions and the ITGF's of the quantum mechanical systems defined above. The results of [26] then tell us that from the ITGF's a unique KMS state for the infinite quantum mechanical system can be reconstructed by analytic continuation in the time variables to the real axis. 
(2) Extensions of Theorem QM to systems with kinetic energies that depend in a non-trivial way on the generalized charges are possible, provided the kinetic energy of one particle is still the generator of a transition function for a Markov process, so that a generalized form of the Feynman-Kac formula (1.18) applies.

(3) For previous constructions of the thermodynamic limit of the pressure see [25] and Refs. given there, and [21] for the case of the Coulomb (matter) system in three dimensions.

Existence theorems for the correlation functions and ITGF's of quantum mechanical systems with correct (Fermi and (or) Bose) statistics have previously been obtained for various classes of short range potentials and small $z$ and $\beta$ in $[12,3]$. In particular, the matter system in the plasma phase (small $z$ and $\beta$ ) with Coulomb - replaced by Yukawa-potentials has been treated in [3]. In a subsequent article we prove such a result for two component systems with Bose-Einstein statistics and two body potentials of positive type at all values of $z$ and $\beta$ at which the systems are stable.

(4) More detailed properties of the pressure (all standard thermodynamic properties) and the correlation functions (monotonicity properties in $z$ and $\beta$ ) in the thermodynamic limit constructed in Theorems C and QM are studied in Sections 4 and 5. In particular, we obtain cluster properties of the Gibbs equilibrium state in the thermodynamic limit for a certain class of potentials. We sketch extensions of our results to potentials with logarithmic singularities at $\left|x-x^{\prime}\right|=0[6]$ and prove the existence of the thermodynamic limit of the pressure for potentials that include positive hard cores.

\section{Statistical Mechanics and Gaussian Integrals}

\section{1. (Formal) Gaussian Integrals}

In this section we briefly recall a by now well known connection between classical statistical mechanics and Gaussian integrals. ${ }^{1}$ Our purpose is to express $\exp \left[-\beta U\left((q)_{N},(x)_{N}\right)\right]$ in terms of a Gaussian integral in case $U\left((q)_{N},(x)_{N}\right)$ is given by (1.5). This then leads to expressions for all the correlation functions in terms of functional integrals. In the classical case this framework has been discussed in detail in $[1,6,29]$.

Let the potential $V$ be some function on $\left(Q \times \mathbb{R}^{v}\right)^{\times 2}$ of positive type satisfying conditions (1.2) and (1.4).

We define a Hilbert space $\mathscr{H}=L^{2}\left(Q \times \mathbb{R}^{v}, d \lambda(q) d^{v} x\right)$ and choose a selfadjoint operator $H$ on $\mathscr{H}$ with $H \geq 1$ and such that, for some $n<\infty, H^{-n}$ is HilbertSchmidt. We let $\mathscr{H}^{k}$ be (the completion of) $D\left(H^{k / 2}\right)$ in the norm $\left\|H^{k / 2} f\right\|, f \in \mathscr{H}$, $k=\ldots-2,-1,0,1,2, \ldots$. Then $\mathscr{S}$ denotes all real functions in $\mathscr{H}^{\infty}$ and $\mathscr{S}^{\prime}$ all real elements of $\mathscr{H}^{-\infty} ; \mathscr{S}$ is a nuclear space. Vectors in $\mathscr{S}$ are denoted by $f, g, \ldots$ and vectors in $\mathscr{S}^{\prime}$ by $\phi, \psi, \chi, \ldots$. Let

$$
\langle f, V g\rangle=\int d \lambda(q) d \lambda\left(q^{\prime}\right) d^{v} x d^{v} x^{\prime} \bar{f}(q, x) V\left(q, x ; q^{\prime}, x^{\prime}\right) g\left(q^{\prime}, x^{\prime}\right) .
$$

1 See "Note Added" and Ref. [29] 
We choose $H$ such that $\mathscr{S}$ is in the quadratic form domain of $V$, and $V$ is continuous on $\mathscr{S} \times \mathscr{S}$, hence on $\mathscr{S} \otimes \mathscr{S}$, (by nuclearity).

Consider now the functional

$$
Z_{V}(f)=e^{-(1 / 2)\langle f, V f\rangle} \text { on } \mathscr{S} \text {. }
$$

This is the characteristic functional of a Gaussian measure $d \mu_{V}$ with mean 0 and covariance $V$ and with support contained in $\mathscr{S}^{\prime}$; (Minlos' theorem).

For $\phi \in \mathscr{S}^{\prime}, f \in \mathscr{S}$, let $\phi(f)$ denote the value of $\phi$ at $f$. We define Wick ordering by

$$
: e^{i \phi(f)}:=Z_{V}(f)^{-1} e^{i \phi(f)} \text {. }
$$

The expectation with respect to $d \mu_{V}$ is defined by

$$
\langle F\rangle_{V}=\int_{\mathscr{S}^{\prime}} d \mu_{V}(\phi) F(\phi)
$$

for arbitrary $F \in L^{1}\left(\mathscr{S}^{\prime}, d \mu_{V}\right)$.

Using (2.2)-(2.4) we get

$$
\left\langle\prod_{j=1}^{n}: e^{i \phi\left(f_{j}\right)}:\right\rangle_{V}=e^{-\sum_{1 \leq i<j \leq n}\left\langle f_{i}, V f_{j}\right\rangle}
$$

Assuming temporarily that $V\left(q, x ; q^{\prime}, x^{\prime}\right)$ is continuous in $(q, x)$ and $\left(q^{\prime}, x^{\prime}\right)$ we may let $f_{j}$ tend to $\beta^{1 / 2} \delta_{\left(q_{j}, x_{j}\right)}, j=1, \ldots, n$, and obtain

$$
\left\langle\prod_{j=1}^{n}: e^{i \beta^{1 / 2} \phi\left(q_{j}, x_{j}\right)}:\right\rangle_{V}=e^{-\sum_{1 \leq i<j \leq n} \beta V\left(q_{i}, x_{i} ; q_{j}, x_{j}\right)}=e^{-\beta U\left((q)_{N},(x)_{N}\right)} .
$$

If we impose condition (1.4), i.e.

$$
V\left(q, x ; q^{\prime}, x^{\prime}\right)=-V\left(-q, x ; q^{\prime}, x^{\prime}\right)=-V\left(q, x ;-q^{\prime}, x^{\prime}\right)
$$

we conclude that

$$
\left\langle\prod_{j=1}^{n}: e^{i \beta^{1 / 2} \varepsilon_{j} \phi\left(q_{j}, x_{j}\right)}:\right\rangle_{V}=\exp \left[-\sum_{1 \leq i<j \leq n} \beta V\left(\varepsilon_{i} q_{i}, x_{i} ; \varepsilon_{j} q_{j}, x_{j}\right)\right],
$$

with $\varepsilon_{j}= \pm 1$, for all $j=1, \ldots, n$.

We define

$$
C_{\Lambda}^{\beta}=\int_{Q} d \lambda(q) \int_{\Lambda} d^{v} x: e^{i \beta^{1 / 2} \phi(q, x)}:
$$

Using (2.7) and the symmetry $d \lambda(q)=d \lambda(-q)$ - see (1.4) — we see that

$$
C_{\Lambda}^{\beta}=\int_{Q} d \lambda(q) \int_{\Lambda} d^{v} x: \cos \beta^{1 / 2} \phi(q, x):
$$

$\mu_{V}$-almost every where.

Note that

$$
\begin{aligned}
& \frac{1}{2}\left(: e^{i \beta^{1 / 2} \phi(q, x)}:+: e^{i \beta^{1 / 2} \phi(-q, x)}:\right) \\
& \quad=e^{\beta / 2 V(q, x ; q, x)} \cos \left[\beta^{1 / 2} \phi(q, x)\right]=: \cos \beta^{1 / 2} \phi(q, x):
\end{aligned}
$$


From definition (1.6) of $\Xi_{\Lambda}(\beta, z),(2.6)$ and (2.8)-(2.10) we conclude that

$$
\Xi_{\Lambda}(\beta, z)=\sum_{N=0}^{\infty} \frac{z^{N}}{N !}\left\langle\left[C_{\Lambda}^{\beta}\right]^{N}\right\rangle_{V}=\left\langle e^{z C_{\Lambda}^{\beta}}\right\rangle_{V},
$$

and from (1.8), (2.6) and (2.8)-(2.10)

$$
\begin{aligned}
\rho_{\Lambda}\left(\beta, z ;(q)_{N},(x)_{N}\right) & =\Xi_{\Lambda}(\beta, z)^{-1}\left\langle\prod_{j=1}^{N} z: e^{i \beta^{1 / 2} \phi\left(q_{j}, x_{j}\right)}: e^{z C_{A}^{\beta}}\right\rangle_{V} \\
& \equiv\left\langle\prod_{j=1}^{N} z: e^{i \beta^{1 / 2} \phi\left(q_{j}, x_{j}\right)}:\right\rangle_{\Lambda}(\beta, z) .
\end{aligned}
$$

In order to extend these formulas to the quantum case we combine Gaussian integrals with the Wiener measure. Let

$$
\mathbb{V}\left(q, x, \tau ; q^{\prime}, x^{\prime}, \tau^{\prime}\right)=V\left(q, x ; q^{\prime}, x^{\prime}\right) \delta\left(\tau-\tau^{\prime}\right)
$$

where $\tau$ and $\tau^{\prime}$ are in the interval $[0, \beta]$ (conveniently viewed as a circle of circumference $\beta$ ).

Let $\mathscr{H}=L^{2}\left(Q \times \mathbb{R}^{v} \times[0, \beta], d \lambda(q) d^{v} x d \tau\right)$. As above we introduce $H,\left\{\mathscr{H}^{k}\right\}_{k \in \mathbb{Z}}$, $\mathscr{S}$ and $\mathscr{S}^{\prime}$ and define a Gaussian measure $d \mu_{\vee}$ on $\mathscr{S}^{\prime}$ with mean 0 and covariance $\mathbb{V}$, the characteristic functional of which is given by

$$
Z_{\mathbb{v}}(f)=e^{-(1 / 2)\langle f, \vee f\rangle}, f \in \mathscr{S} .
$$

Then

$$
\left\langle\prod_{j=1}^{N}: e^{i \phi\left(f_{j}\right)}:\right\rangle_{v}=e^{-\sum_{1 \leq i<j \leq n}\left\langle f_{i}, \vee f_{j}\right\rangle}
$$

where Wick ordering is given by (2.3) with $Z_{V}$ replaced by $Z_{\mathbb{V}}$.

For $f_{j}(\bar{q}, x, \tau)=\delta\left(\bar{q}-q_{j}\right) \delta\left(x-\omega_{j}(\tau)\right)$ we obtain

$$
\begin{aligned}
& \left\langle\prod_{j=1}^{N}: e^{i \int_{0}^{B} d \tau \phi\left(q_{j}, \omega_{j}(\tau), \tau\right)}:\right\rangle \\
& =e^{-\int_{0}^{B} d \tau U\left((q)_{N},(\omega(\tau))_{N}\right)} .
\end{aligned}
$$

We set

$$
\mathbb{C}_{\Lambda}^{\beta}=\int_{Q} d \lambda(q) \int_{\Lambda} d^{v} x \int_{\Omega} \operatorname{Pr}_{\Lambda}^{\beta}(q, x, x ; d \omega): \cos _{0}^{\beta} d \tau \phi(q, \omega(\tau), \tau):
$$

Using (1.14) and (1.18) and repeating the arguments (2.8) - (2.10) given in the classical case we find

$$
\Xi_{\Lambda}(\beta, z)=\left\langle e^{z \mathbb{C} \beta_{\Lambda}}\right\rangle_{V}
$$

(which is seen by expanding the exponential in a power series and applying (2.15), (2.14), (1.18) and (1.14)).

Moreover the density correlation functions are given by

$$
\begin{aligned}
& \rho_{\Lambda}\left(\beta, z ;(q)_{N},(x)_{N}\right)=\Xi_{\Lambda}(\beta, z)^{-1} \\
& \quad \times z^{N}\left\langle\prod_{j=1}^{N} \int \operatorname{Pr}_{\Lambda}^{\beta}\left(q_{j}, x_{j}, x_{j} ; d \omega_{j}\right) e^{i \int_{0}^{\beta} d \tau \phi\left(q_{j}, \omega_{j}(\tau), \tau\right)} e^{z \mathbb{C}_{\Lambda}}\right\rangle_{V} .
\end{aligned}
$$


Similar, somewhat more complicated expression for all the correlation functions and the ITGF's are derived in Appendix 1.

\subsection{Estimates}

In this section we propose to justify the somewhat formal expressions for the partition function and the correlation functions found in Section 2.1. This is done by means of some very simple estimates. Indeed, the following are the main analytical estimates of this paper: As functions on $\mathscr{S}^{\prime}$

$$
\left|: e^{i a \phi(q, x)}:\right|=e^{\left(a^{2} / 2\right) V(q, x ; q, x)},
$$

and

$$
\begin{aligned}
& \left|: \mathrm{e}^{i a \int_{0}^{\beta} d \tau \phi(q, \omega(\tau), \tau)}:\right| \\
& \quad \leq e^{\left(a^{2} / 2\right) \int_{0}^{\beta} d \tau V(q, \omega(\tau) ; q, \omega(\tau))} \\
& \quad \leq \frac{1}{\beta} \int_{0}^{\beta} d \tau e^{\left(\beta a^{2} / 2\right) V(q, \omega(\tau) ; q, \omega(\tau))},
\end{aligned}
$$

by Jensen's inequality.

Since we have assumed that

$$
K_{\alpha} \equiv \sup _{x \in \mathbb{R}^{\nu}} \int_{\mathcal{Q}} d \lambda(q) e^{(\alpha / 2) V(q, x ; q, x)}
$$

be finite, for all $\alpha>0$, we conclude that in the classical case

$$
\begin{aligned}
\left|C_{\Lambda}^{\beta}\right| & \leq \int_{Q} d \lambda(q) \int_{\Lambda} d^{v} x\left|: \cos \beta^{1 / 2} \phi(q, x):\right| \leq \int_{\Lambda} d^{v} x \int_{Q} d \lambda(q) e^{(\beta / 2) V(q, x ; q, x)} \\
& \leq K_{\beta}|\Lambda|<\infty, \text { and }
\end{aligned}
$$

in the quantum case

$$
\begin{aligned}
\left|\mathbb{C}_{\Lambda}^{\beta}\right| & \leq \int_{Q} d \lambda(q) \int_{\Lambda} d^{v} x \int_{\Omega} \operatorname{Pr}_{\Lambda}^{\beta}(q, x, x ; d \omega)\left|: \cos \int_{0}^{\beta} d \tau \phi(q, \omega(\tau), \tau):\right| \\
& \leq \int_{\Lambda} d^{v} x\left\{\sup _{q \in Q} \int_{\Omega} \operatorname{Pr}_{\Lambda}^{\beta}(q, x, x ; d \omega)\right\} \int_{Q} d \lambda(q) e^{(1 / 2) \int_{0}^{\beta} V(q, \omega(\tau), q, \omega(\tau)) d \tau} \\
& \leq \int_{\Lambda} d^{v} x \sup _{q \in Q} \int_{\Omega} \operatorname{Pr}_{\mathbb{R}^{\nu}}^{\beta}(q, x, x ; d \omega) K_{\beta} \\
& =K_{\beta} e^{-\beta p^{2} / 2 \bar{m}} d^{v} p|\Lambda| \\
& =K_{\beta}\left(\frac{2 \pi \bar{m}}{\beta}\right)^{v / 2}|\Lambda|
\end{aligned}
$$

inequality (2.22) follows from inequality (2.20) and the simple fact that

$$
\operatorname{Pr}_{\Lambda}^{\beta}(q, x, y ; d \omega) \leq \operatorname{Pr}_{\mathbb{R}}^{\beta}(q, x, y ; d \omega) ;
$$


moreover

$$
\int_{\Omega} \operatorname{Pr}_{\mathbb{R}^{\nu}}^{\beta}(q, x, y ; d \omega)=\int e^{i p(x-y)} e^{-\beta p^{2} / 2 m(q)} d^{v} p
$$

which, for $x=y$, gives (2.23).

We set $z \equiv \zeta$, in the classical case, and $z \equiv \zeta\left(\frac{2 \pi \bar{m}}{\beta}\right)^{-v / 2}$, in the quantum case. Then

$$
\left|\Xi_{A}(\beta, z)\right| \leq\left\langle\left|e^{z C_{A}^{\beta}}\right|\right\rangle_{V} \leq\left\langle e^{\left|\operatorname{Re} z C_{A}^{\beta}\right|}\right\rangle_{V} \leq e^{|\operatorname{Re} \zeta| K_{\beta}|\Lambda|},
$$

and this extends to the quantum case; see (2.6) and (2.23).

We summarize our estimates in

Theorem 2.1. Under the hypotheses of Theorems $C$ and $Q M$ the following estimates hold:

(1) For all complex $z$

$$
\left|\Xi_{\Lambda}(\beta, z)\right| \leq e^{|\operatorname{Re} \zeta| K_{\beta}|\Lambda|} .
$$

(2) In the classical case,

$$
\mid \rho_{\Lambda}\left(\beta, z ;(q)_{N},\left.(x)_{N}|\leq| \zeta\right|^{N} e^{(\beta / 2) \sum_{i=1}^{N} V\left(q_{i}, x_{i} ; q_{i}, x_{i}\right)}\right.
$$

for all real $z$ and $\beta \geq 0$.

(3) In the quantum mechanical case, and for all real $z$ and $\beta \geq 0$

$$
\begin{aligned}
& \left|\rho_{\Lambda}\left(\beta, z ;(q)_{N},(x)_{N}\right)\right| \leq|\zeta|^{N}\left(\frac{2 \pi \bar{m}}{\beta}\right)^{-N v / 2} \\
& \cdot \int_{\Omega^{N}} \prod_{j=1}^{N} \operatorname{Pr}_{\mathbb{R}^{v}}^{\beta}\left(q_{j}, x_{j}, x_{j} ; d \omega_{j}\right) \\
& \cdot e^{(1 / 2) \sum_{j=1}^{N} \int_{0}^{\beta} V\left(q_{j}, \omega_{j}(\tau), q_{j}, \omega_{j}(\tau)\right) d \tau} \\
& \leq|\zeta|^{N} e^{(\beta / 2) \sum_{j=1}^{N} \sup _{x} V\left(q_{j}, x ; q_{j}, x\right)} .
\end{aligned}
$$

The proof of (2) follows directly from (1), (2.12) and (2.18), and the one of (3) from (1), (2.17), (2.20) and (2.24).

Remarks. 1. This theorem justifies all formulas of Section 2.1 completely.

2. Theorem 2.1, (1) immediately extends to the quantum mechanical system with two body potential V and Fermi statistics. In the case of Bose statistics it remains true for small enough $|z|$, depending on $K_{\beta}$. These results follow from the classical result by means of the Golden-Thompson inequality, as explained in [6, Section 4]. 3. In Appendix 1 we extend the uniform bounds of Theorem 2.1, (3) to arbitary correlation functions and ITGF's.

4. In a subsequent article the methods of Section 2 are extended to quantum mechanical systems with Fermi-or Bose-Einstein statistics, for all positive 
values of $z$ and $\beta$ for which stability holds; see Remark 2. Theorem 2.1, (3) and this result appear to be new.

5. The bounds of Theorem 2.1 are very crude. In certain situations much finer estimates can be obtained; see $[3,6,25]$.

\section{Conditioning - and Correlation Inequalities}

Let $\mathscr{H}$ be a real Hilbert space, and let $C$ be a bounded positive quadrative form on $\mathscr{H}$. Let $\phi$ be the (unique) Gaussian process with mean 0 and covariance $C$ indexed by $\mathscr{H}$. The associated Gaussian measure with mean 0 and covariance $C$ (defined on some measure space $\left(\mathscr{S}^{\prime}, \Sigma\right)$ ) is denoted $d \mu_{c} ;$ (see e.g. [23]).

Let $(X, S)$ be some measure space and $d \rho$ a positive, finite measure on $X$. We choose a measurable mapping

$x \longmapsto l_{x} \in \mathscr{H}$

from $X$ to $\mathscr{H}$ such that $\int_{X} d \rho(x) e^{(1 / 2) C\left(l_{x}, l_{x}\right)}$ is finite.

A partition function is defined by

$$
\Xi(C, \rho) \equiv \Xi(\rho)=\int_{\mathscr{S}^{\prime}} d \mu_{C}(\phi) \exp \left\{\int_{X} d \rho(x) \cos \phi\left(l_{x}\right)\right\} .
$$

For $F \in L^{1}\left(\mathscr{S}^{\prime}, d \mu_{\mathrm{C}}\right)$, we set

$$
\langle F\rangle_{C, \rho} \equiv\langle F\rangle_{\rho}=\Xi(\rho)^{-1} \int_{\mathscr{S}^{\prime}} d \mu_{C}(\phi) F(\phi) \exp \left\{\int_{X} d \rho(x) \cos \phi\left(l_{x}\right)\right\} .
$$

In the following, $m, n, l, g, \ldots$ always denote vectors in $\mathscr{H}$.

The main result of Section 3 is

Theorem 3.1. (1) Let $\rho_{1}$ and $\rho_{2}$ be positive, finite measures on $(X, S)$. Then

$$
\Xi\left(\rho_{1}+\rho_{2}\right) \geq \Xi\left(\rho_{1}\right) \cdot \Xi\left(\rho_{2}\right) .
$$

(2) (Conditioning) Let $0 \leq C_{1} \leq C_{2}$ and define

$d \rho_{12}(x)=e^{(1 / 2)\left(C_{2}\left(l_{x}, l_{x}\right)-C_{1}\left(l_{x}, l_{x}\right)\right)} d \rho(x)$.

Then

$\Xi\left(C_{1}, \rho\right) \leq \Xi\left(C_{2}, \rho_{12}\right)$.

(3) (Inverse conditioning). Let $0 \leq C_{1} \leq C_{2}$.

Then

$\Xi\left(C_{2}, \rho\right) \leq \Xi\left(C_{1}, \rho\right)$

(4) $\left\langle\prod_{j=1}^{k} \cos \phi\left(m_{j}\right)\right\rangle_{\rho} \geq 0$

(5) $\left\langle\prod_{j=1}^{k} \cos \phi\left(m_{j}\right) \prod_{j=1}^{l} \cos \phi\left(n_{j}\right)\right\rangle_{\rho}-\left\langle\prod_{j=1}^{k} \cos \phi\left(m_{j}\right)\right\rangle_{\rho}\left\langle\prod_{j=1}^{l} \cos \phi\left(n_{j}\right)\right\rangle_{\rho} \geq 0$.

Remarks. Inequality (1) appears to be new; a slightly more general version of 
the conditioning inequality (2) has been proven in [6] and is a consequence of a general observation in [14]. A proof of the inverse conditioning inequality (3) has been given in [6], but it has probably been known by various people for a long time. Inequality (4) is trivial. Inequality (5) is a somewhat more general version of Park's inequality, [24]. An extension of inequality (5) is presented in [9] and applied there to construct a Euclidean invariant infinite volume limit for the "bosonized" form $[4,10]$ of the Yukawa model and massive QED in two spacetime dimensions.

Proof. The basic ideas (due to Ginibre [13]) of the proof of Theorem 3.1 are

1. Duplicate variables, [13]

2. trigonometric identities [13], namely

(3.a) $e^{i \alpha}=\cos \alpha+i \sin \alpha$

(3.b) $\cos (\alpha \pm \beta)=\cos \alpha \cos \beta \mp \sin \alpha \sin \beta$

(3.c) $\cos \alpha \pm \cos \beta=2\left\{\begin{array}{l}\cos \left(\frac{\alpha+\beta}{2}\right) \cos \left(\frac{\alpha-\beta}{2}\right) \\ \sin \left(\frac{\alpha+\beta}{2}\right) \sin \left(\frac{\beta-\alpha}{2}\right)\end{array}\right.$

(3.d) $\cos \alpha \cos \beta=\frac{1}{2}[\cos (\alpha+\beta)+\cos (\alpha-\beta)]$

(3.e) $\prod_{j=1}^{k} \cos \alpha_{j}=\left(\frac{1}{2}\right)^{k} \sum_{\left\{e_{j}\right\}} \cos \left(\sum_{j=1}^{k} e_{j} \alpha_{j}\right)$

with $e_{j}= \pm 1, j=1,2, \ldots, k$.

We note that (3.e) follows from $\cos \alpha=\cos (-\alpha)$ and (3.d), by induction.

Proof of $(I)$. Let $\phi_{1}, \phi_{2}$ be two independent, equivalent Gaussian processes with mean 0 and covariance $C$. Then

$$
\begin{aligned}
\Xi\left(\rho_{1}+\rho_{2}\right)-\Xi\left(\rho_{1}\right) \Xi\left(\rho_{2}\right)= & \int d \mu_{c}\left(\phi_{1}\right) d \mu_{c}\left(\phi_{2}\right) e^{\int d \rho_{1}(x) \cos \phi_{1}\left(l_{x}\right)} \\
& \cdot\left[e^{\int d \rho_{2}(x) \cos \phi_{1}\left(l_{x}\right)}-e^{\int d \rho_{2}(x) \cos \phi_{2}\left(l_{x}\right)}\right] .
\end{aligned}
$$

(This is the duplicate variable method of [13], see also [24]).

Let $\vec{\phi}=\left(\phi_{1}, \phi_{2}\right)$ be the Gaussian process with mean 0 and covariance $\mathbb{C}$ given by $\mathbb{C}_{i j}=\delta_{i j} C$, so that

$d \mu_{\mathbb{C}}(\vec{\phi})=d \mu_{c}\left(\phi_{1}\right) d \mu_{c}\left(\phi_{2}\right)$.

Clearly, $R^{T} \mathbb{C} R=\mathbb{C}$, for all orthogonal transformations $R$ of "field space" $\mathbb{R}^{2}$. Hence

$$
d \mu_{\mathbb{C}}(\vec{\phi})=d \mu_{\mathbb{C}}(R \vec{\phi}) \text {, for all } R \in 0(2) .
$$


Let

$$
\left.\begin{array}{l}
\psi=(R \vec{\phi})_{1}=\frac{\phi_{1}+\phi_{2}}{\sqrt{2}} \\
\chi=(R \vec{\phi})_{2}=\frac{-\phi_{1}+\phi_{2}}{\sqrt{2}}
\end{array}\right\} .
$$

This is an orthogonal transformation of field space, and therefore

$$
d \mu_{c}\left(\phi_{1}\right) d \mu_{C}\left(\phi_{2}\right)=d \mu_{C}(\psi) d \mu_{c}(\chi) \text {. }
$$

The inverse of (3.6) is given by

$$
\phi_{1}=\frac{\psi-\chi}{\sqrt{2}}, \phi_{2}=\frac{\psi+\chi}{\sqrt{2}} .
$$

Let

$$
\psi_{x}=\frac{\psi\left(l_{x}\right)}{\sqrt{2}} \text { and } \chi_{x}=\frac{\chi\left(l_{x}\right)}{\sqrt{2}}
$$

Using (3.4) and (3.7)-(3.9) we obtain

$$
\begin{aligned}
\Xi\left(\rho_{1}+\rho_{2}\right)-\Xi\left(\rho_{1}\right) \Xi\left(\rho_{2}\right)= & \int d \mu_{C}(\psi) d \mu_{C}(\chi) e^{\int\left(d \rho_{1}+d \rho_{2}\right)(x) \cos \psi_{x} \cos \chi_{x}} \\
& \cdot e^{\int d \rho_{1}(x) \sin \psi_{x} \sin \chi_{x}} 2 \sinh \left(\int d \rho_{2}(x) \sin \psi_{x} \sin \chi_{x}\right) .
\end{aligned}
$$

Expanding the exponentials and the hyperbolic sine in power series we get

$$
\begin{aligned}
\Xi\left(\rho_{1}+\rho_{2}\right)-\Xi\left(\rho_{1}\right) \Xi\left(\rho_{2}\right)= & 2 \sum_{n_{1}, n_{2}, n_{3}=0}^{\infty} \frac{1}{n_{1} ! n_{2} !\left(2 n_{3}+1\right) !} \prod_{j=1}^{n_{1}} \int\left(d \rho_{1}+d \rho_{2}\right)\left(x_{j}\right) \\
& \cdot \prod_{j=1}^{n_{2}} \int d \rho_{1}\left(x_{j}^{\prime}\right) \prod_{j=1}^{2 n_{3}+1} \int d \rho_{2}\left(x_{j}^{\prime \prime}\right) \\
& \cdot \int d \mu_{C}(\psi) d \mu_{c}(\chi) \prod_{j=1}^{n_{1}}\left(\cos \psi_{x_{j}} \cos \chi_{x_{j}}\right) \\
& \cdot \prod_{j=1}^{n_{2}}\left(\sin \psi_{x_{j}^{\prime}} \sin \chi_{x_{j}^{\prime}}\right) \prod_{j=1}^{2 n_{3}+1}\left(\sin \psi_{x_{j}^{\prime \prime}} \sin \chi_{x_{j}^{\prime \prime}}\right) .
\end{aligned}
$$

The $d \mu_{C}(\psi) d \mu_{C}(\chi)$-integral on the r.h.s. of (3.10) is

$$
\left|\int d \mu_{C}(\psi) \prod_{j=1}^{n_{1}} \cos \psi_{x_{j}} \prod_{j=1}^{n_{2}} \sin \psi_{x_{j}^{\prime}} \prod_{j=1}^{2 n_{3}+1} \sin \psi_{x_{j}^{\prime \prime}}\right|^{2} \geq 0
$$

and this completes the proof of (1).

Proof of (2). Let $\phi_{1 / 2}$ be the Gaussian process with mean 0 and covariance $C_{1 / 2}$ and $\phi_{1}^{\prime}$ the one with mean 0 and covariance $C_{2}-C_{1} \geq 0$. Then

$$
\int d \mu_{C_{1}}\left(\phi_{1}\right) d \mu_{C_{2}-C_{1}}\left(\phi_{1}^{\prime}\right) F\left(\phi_{1}+\phi_{1}^{\prime}\right)=\int d \mu_{C_{2}}\left(\phi_{2}\right) F\left(\phi_{2}\right)
$$

for all $d \mu_{C_{2}}$-integrable functions $F$. To see this, it suffices to take $F(\phi)=\exp i \phi(g)$, 
$g \in \mathscr{H}$, for which (3.11) is obvious. Thus

$$
\begin{aligned}
\Xi\left(C_{2}, \rho_{12}\right)= & \int d \mu_{C_{2}}\left(\phi_{2}\right) e^{\int d \rho_{12}(x) \cos \phi_{2}\left(l_{x}\right)}=\int d \mu_{C_{1}}\left(\phi_{1}\right) d \mu_{C_{2}-C_{1}}\left(\phi_{1}^{\prime}\right) \exp \left[\int d \rho_{12}(x)\right. \\
& \left.\cdot\left\{\cos \phi_{1}\left(l_{x}\right) \cos \phi_{1}^{\prime}\left(l_{x}\right)-\sin \phi_{1}\left(l_{x}\right) \sin \phi_{1}^{\prime}\left(l_{x}\right)\right\}\right] \\
\geq & \int d \mu_{C_{1}}\left(\phi_{1}\right) \exp \left[\int d \mu_{C_{2}-C_{1}}\left(\phi_{1}^{\prime}\right) \int d \rho_{12}(x)\right. \\
& \left.\cdot\left\{\cos \phi_{1}\left(l_{x}\right) \cos \phi_{1}^{\prime}\left(l_{x}\right)-\sin \phi_{1}\left(l_{x}\right) \sin \phi_{1}^{\prime}\left(l_{x}\right)\right\}\right] \\
= & \int d \mu_{C_{1}}\left(\phi_{1}\right) \exp \left[\int d \rho_{12}(x) e^{-(1 / 2)\left(C_{2}\left(l_{x}, l_{x}\right)-C_{1}\left(l_{x}, l_{x}\right)\right)} \cos \phi_{1}\left(l_{x}\right)\right] \\
= & \Xi\left(C_{1}, \rho\right), \text { which is }(2) .
\end{aligned}
$$

The equation before (3.12) follows from (3.11) and (3.b), and inequality (3.12) is a consequence of Jensen's inequality.

For the proof of (3) see Corollary 3.2, or [6].

Proof of (4). Obviously it suffices to show that

$$
\sum_{n=0}^{\infty} \frac{1}{n !} \prod_{j=1}^{n} \int d \rho\left(x_{j}\right) \int d \mu_{c}(\phi) \prod_{i=1}^{k} \cos \phi\left(m_{i}\right) \prod_{j=1}^{n} \cos \phi\left(l_{x_{j}}\right) \geq 0 .
$$

Using (3.e) we see that this follows from

$$
\int d \mu_{C}(\phi) \cos \phi(h)=e^{-(1 / 2)(h, C h)}>0,
$$

for all $h \in \mathscr{H} ;(h, C h) \equiv C(h, h)$.

Proof of (5). By (3.e) and the linearity of $\langle\longrightarrow\rangle_{\rho}$ it suffices to show

$\langle\cos \phi(m) \cos \phi(n)\rangle_{\rho}-\langle\cos \phi(m)\rangle_{\rho}\langle\cos \phi(n)\rangle_{\rho} \geq 0$.

Using duplicate variables the 1.h.s. of (3.13) can be written as

$$
\begin{aligned}
& \Xi(\rho)^{-2} \int d \mu_{C}\left(\phi_{1}\right) d \mu_{C}\left(\phi_{2}\right)\left\{\cos \phi_{1}(m)\right\} \\
& \quad \cdot\left[\cos \phi_{1}(n)-\cos \phi_{2}(n)\right] \exp \int d \rho(x)\left(\cos \phi_{1}\left(l_{x}\right)+\cos \phi_{2}\left(l_{x}\right)\right) \\
& =\Xi(\rho)^{-2} \sum_{n=0}^{\infty} \frac{1}{n !} \prod_{j=1}^{n} \int d \rho\left(x_{j}\right) \int d \mu_{C}(\psi) \\
& \cdot d \mu_{C}(\chi)\left\{\cos \frac{\psi(m)}{\sqrt{2}} \cos \frac{\chi(m)}{\sqrt{2}}+\sin \frac{\psi(m)}{\sqrt{2}} \sin \frac{\chi(m)}{\sqrt{2}}\right\}\left[2 \sin \frac{\psi(n)}{\sqrt{2}} \sin \frac{\chi(n)}{\sqrt{2}}\right] \\
& \quad: \prod_{j=1}^{n}\left(2 \cos \psi_{x_{j}} \cos \chi_{x_{j}}\right) .
\end{aligned}
$$

This equation follows by inserting (3.7)-(3.8) and applying (3.b) to $\{\ldots$.$\} ,$ (3.c) to $[\ldots .$.$] and to (...) and expanding the exponential. As in the proof of (1)$ one sees that each term in the series on the r.h.s. of (3.14) is positive.

Q.E.D

Remark: Using (3.a) and the equation $d \mu_{c}(\phi)=d \mu_{C}(-\phi)$, we get

$$
\begin{aligned}
& \left\langle e^{i \phi(m)}\right\rangle_{\rho} \geq 0, \\
& \left\langle e^{i \phi(m)} \cos \phi(n)\right\rangle_{\rho}-\left\langle e^{i \phi(m)}\right\rangle_{\rho}\langle\cos \phi(n)\rangle_{\rho} \geq 0 ;
\end{aligned}
$$


this is essentially the form of the inequalities found in [24].

We emphasize that most of the ideas used in the proof of Theorem 3.1 are already in Ginibre's basic paper [13]; our applications are however new. Finally we note that Theorem 3.1,(1) can also be obtained directly from Theorem 3.1,(5) by expanding the exponential!

Corollary 3.2. For $\rho_{1} \leq \rho_{2}$ and $C_{1}^{-1} \leq C_{2}^{-1}$ (or, equivalently, $C_{2} \leq C_{1}$ ),

(1) $\left\langle\prod_{j=1}^{k} \cos \phi\left(m_{j}\right)\right\rangle_{c_{1}, \rho_{1}} \leq\left\langle\prod_{j=1}^{k} \cos \phi\left(m_{j}\right)\right\rangle_{C_{2}, \rho_{2}}$.

(2) $\left\langle\phi(m)^{2}\right\rangle_{C_{2}, \rho_{2}} \leq\left\langle\phi(m)^{2}\right\rangle_{C_{1}, \rho_{1}} \leq\left(m, C_{1} m\right)$.

(3) Moreover, $\Xi\left(C_{1}, \rho_{1}\right) \leq \Xi\left(C_{2}, \rho_{2}\right) ;\left(\right.$ for $\rho_{1}=\rho_{2}=\rho$ this is Theorem 3.1, (3)).

Proof. From Theorem 3.1, (5) we get

$$
\begin{aligned}
\frac{1}{\varepsilon^{2}} & \langle(1-\cos \varepsilon \phi(m)) \cos \phi(n)\rangle_{\rho} \\
& \left.-\langle 1-\cos \varepsilon \phi(m)\rangle_{\rho}\langle\cos \phi(n)\rangle_{\rho}\right\} \leq 0 .
\end{aligned}
$$

As $\varepsilon$ tends to 0 this yields

$$
\left\langle\phi(m)^{2} \cos \phi(n)\right\rangle_{\rho}-\left\langle\phi(m)^{2}\right\rangle_{\rho}\langle\cos \phi(n)\rangle_{\rho} \leq 0
$$

and, by repeating the argument,

$$
\left\langle\phi(m)^{2} \phi(n)^{2}\right\rangle_{\rho}-\left\langle\phi(m)^{2}\right\rangle_{\rho}\left\langle\phi(n)^{2}\right\rangle_{\rho} \geq 0 .
$$

Similar inequalities with $\phi(m)^{2}$ replaced by $(\sin \phi(m))^{2}$ follow by using

$$
(\sin \phi(m))^{2}=\frac{1}{2}-\frac{1}{2} \cos \phi(2 m) \text {. }
$$

We shall apply (3.16) and (3.17) at several places.

Proof of $(l)$. We first show that for $\rho_{1} \leq \rho_{2}$

$$
\left\langle\prod_{j=1}^{k} \cos \phi\left(m_{j}\right)\right\rangle_{\rho_{1}} \leq\left\langle\prod_{j=1}^{k} \cos \phi\left(m_{j}\right)\right\rangle_{\rho_{2}} .
$$

By (3.e) it suffices to show this for $k=1, m_{1}=m$.

Let $\rho_{s}=(1-s) \rho_{1}+s \rho_{2}, s \in[0,1]$. Then

$$
\begin{gathered}
\frac{d}{d s}\langle\cos \phi(m)\rangle_{\rho_{s}}=\int d\left(\rho_{2}-\rho_{1}\right)(x)\left[\left\langle\cos \phi(m) \cos \phi\left(l_{x}\right)\right\rangle_{\rho_{s}}\right. \\
\left.-\langle\cos \phi(m)\rangle_{\rho_{s}}\left\langle\cos \phi\left(l_{x}\right)\right\rangle_{\rho_{s}}\right] \geq 0 \text {, by Theorem 3.1, (5). }
\end{gathered}
$$

Upon integrating (3.19) we get (3.18).

Next, we prove that, for $C_{1}^{-1} \leq C_{2}^{-1}$ (i.e. $C_{2} \leq C_{1}$ ),

$$
\langle\cos \phi(m)\rangle_{C_{1}, \rho} \leq\langle\cos \phi(m)\rangle_{C_{2}, \rho} \text {. }
$$

We temporarily assume that $C_{2}^{-1}-C_{1}^{-1}$ is a finite rank operator on $\mathscr{H}$. 
Let $h_{k}$ be the eigen-vector of $C_{2}^{-1}-C_{1}^{-1}$ corresponding to an eigen-value $\lambda_{k}>0$, $k=1, \ldots, N<\infty$.

Let $C_{s}=\left((1-s) C_{1}^{-1}+s C_{2}^{-1}\right)^{-1}$, and

$$
Z_{s}=\int d \mu_{C_{1}}(\phi) \exp \left[-\frac{s}{2}\left(\phi,\left(C_{2}^{-1}-C_{1}^{-1}\right) \phi\right)\right] \text {. }
$$

Then

$$
\begin{aligned}
d \mu_{C_{s}}(\phi) & =Z_{s}^{-1} e^{-(s / 2)\left(\phi,\left(C_{2}^{-1}-C_{1}^{-1}\right) \phi\right)} d \mu_{C_{1}}(\phi) \\
& =Z_{s}^{-1} e^{-(s / 2) \sum_{k=1}^{N} \lambda_{k} \phi\left(h_{k}\right)^{2}} d \mu_{C_{1}}(\phi) .
\end{aligned}
$$

Therefore

$$
\begin{aligned}
& \frac{d}{d s}\langle\cos \phi(m)\rangle_{C_{s}, \rho}=-\frac{1}{2} \sum_{k=1}^{N} \lambda_{k}\left\{\left\langle\cos \phi(m) \phi\left(h_{k}\right)^{2}\right\rangle_{c_{s}, \rho}\right. \\
&\left.\quad-\langle\cos \phi(m)\rangle_{C_{s}, \rho}\left\langle\phi\left(h_{k}\right)^{2}\right\rangle_{c_{s}, \rho}\right\} \\
& \geq 0, \text { by (3.16). }
\end{aligned}
$$

Integration of (3.22) gives (3.20). The general case, i.e. $C_{2}^{-1}-C_{1}^{-1}$ an arbitrary positive, closed quadratic form, follows by a limiting argument. This completes the proof of (1).

Proof of (2). This follows from (1) by using

$$
\begin{aligned}
\phi(m)^{2}= & \lim _{\varepsilon \downarrow 0}\left(1 / \varepsilon^{2}\right)[1-\cos \varepsilon \phi(m)] . \text { Note that } \\
& C_{1}(m, m) \equiv\left(m, C_{1} m\right)=\left\langle\phi(m)^{2}\right\rangle_{C_{1}, \rho=0} .
\end{aligned}
$$

We remark that (2) remains true if we replace $\phi(m)^{2}$ by $(\sin \phi(m))^{2}$ and $(m$, $\left.C_{1} m\right)$ by $\frac{1}{2}\left[1-\exp \left(-4\left(m, C_{1} m\right)\right)\right]$.

Proof of (3). Clearly $\Xi\left(C, \rho_{1}\right) \leq \Xi\left(C, \rho_{2}\right)$ follows from Theorem 3.1, (1), since $\Xi\left(C, \rho_{2}-\rho_{1}\right) \geq 1$.

Let $C_{s}, Z_{s}$ and $d \mu_{C_{s}}$ be defined as in (3.21). Then

$$
\begin{aligned}
\frac{d}{d s} \Xi\left(C_{s}, \rho\right)= & -\frac{1}{2} \sum_{k=1}^{N} \lambda_{k}\left\{\left\langlee^{\int d \rho(x) \cos \phi\left(l_{x}\right)}\right.\right. \\
& \left.\cdot \phi\left(h_{k}\right)^{2}\right\rangle_{C_{s}, 0}-\left\langle e^{\int d \rho(x) \cos \phi\left(l_{\alpha}\right)}\right\rangle_{C_{s}, 0} \\
& \left.\cdot\left\langle\phi\left(h_{k}\right)^{2}\right\rangle_{C_{s}, 0}\right\} \geq 0 .
\end{aligned}
$$

This is seen by expanding the exponential, applying (3.e) and using (3.16). Q.E.D.

Remarks. Theorems $C$ and $Q M$ announced in Section 1 are more or less immediate consequences of the bounds of Section 2.2 and Corollary 3.2. Moreover Corollary 3.2 yields monotonicity in the activity $z$ (and in the measure $d \lambda$ ) and monotonicity properties in the temperature $\beta^{-1}$ for the correlation functions and certain susceptibilities; see Section 4.2. This is useful in the discussion of critical properties of these gases. 


\section{Thermodynamic Limit and Cluster Properties}

\subsection{Proofs of Theorems $C$ and $Q M$}

Proof of Theorem $C,(1)$. In order to apply the inequalities of Section 3 to the proof of Theorem $C$ we must choose

$\left.\begin{array}{l}\quad X=Q \times \mathbb{R}^{v}, \\ \text { and to } l_{x} \text { (defined in (3.1)) there corresponds } \delta_{q} \delta_{x} .\end{array}\right\}$

$$
\text { Let } g_{\Lambda}^{\beta}(q, x)=\exp \left[\frac{\beta}{2} V(q, x ; q, x)\right] \chi_{\Lambda}(x),
$$

(with $\chi_{\Lambda}$ the characteristic function of $\Lambda$ ) and $g^{\beta}=g_{\Lambda=\mathbb{R} v}^{\beta}$. We define

$$
d \rho_{1 / 2}=z g_{\Lambda_{1 / 2}}^{\beta}(q, x) d \lambda(q) d^{v} x, z>0 .
$$

If $\Lambda_{1} \cap \Lambda_{2}=\emptyset$

$$
d\left(\rho_{1}+\rho_{2}\right)=z g_{\Lambda_{1} \cup \Lambda_{2}}^{\beta}(q, x) d \lambda(q) d^{v} x .
$$

We now recall that

$$
: \cos \beta^{1 / 2} \phi(q, x):=g^{\beta}(q, x) \cos \left[\beta^{1 / 2} \phi(q, x)\right] .
$$

See Equations (2.3), (2.10), Section 2.1.

From Equation (2.11), Section 2.1, (4.4) and Theorem 3.1, (1) we obtain

$$
\Xi_{\Lambda_{1} \cup \Lambda_{2}}(\beta, z) \geq \Xi_{\Lambda_{1}}(\beta, z) \Xi_{\Lambda_{2}}(\beta, z),
$$

hence

$$
\log \Xi_{\Lambda_{1} \cup \Lambda_{2}}(\beta, z) \geq \log \Xi_{\Lambda_{1}}(\beta, z)+\log \Xi_{\Lambda_{2}}(\beta, z) .
$$

This inequality asserts superadditivity of $\log \Xi_{\Lambda}(\beta, z)$ in $\Lambda$. Inequality (2.25) and the trivial inequality $\Xi_{\Lambda}(\beta, z) \geq 1$ show that

$$
\frac{1}{|\Lambda|} \log \Xi_{\Lambda}(\beta, z) \text { is bounded uniformly }
$$

in $\Lambda$.

Standard facts about superadditive functions (see e.g. [25]) show now that if $V$ is translation invariant

$$
\lim _{\Lambda \rightarrow \mathbb{R}^{v}} \frac{1}{|\Lambda|} \log \Xi_{\Lambda}(\beta, z)=\lim _{\Lambda \rightarrow \mathbb{R}^{v}} p_{\Lambda}(\beta, z)
$$

exists if $\Lambda$ tends to $\mathbb{R}^{v}$ in the sense of van Hove, [25].

We also claim that the $\operatorname{limit} \lim p_{\Lambda}(\beta, z)$ exists and is independent of $\{\Lambda\}$, whenever $\Lambda \uparrow \mathbb{R}^{v}$, by inclusion. This is seen by considering

$$
\frac{\partial}{\partial z} p_{\Lambda}(\beta, z)=\frac{1}{|\Lambda|} \int_{Q} d \lambda(q) \int_{\Lambda} d^{v} x \rho_{\Lambda}(\beta, z ; q, x)>0 .
$$


We shall prove shortly that, for all $x, z \geq 0$ and $\beta \geq 0$

$$
\lim _{\Lambda \uparrow \mathbb{R}^{v}} \rho_{\Lambda}(\beta, z ; q, x)=\sup _{\Lambda} \rho_{\Lambda}(\beta, z ; q, x)
$$

exists and is independent of $\{\Lambda\}$ if $\Lambda \uparrow \mathbb{R}^{v}$ by inclusion. A standard argument [14] shows then that the same is true for $(\partial / \partial z) p_{\Lambda}(\beta, z)$, for all $z<\infty$, and hence, since

$$
p_{\Lambda}(\beta, z)=\int_{0}^{z} d \zeta p_{\Lambda}(\beta, \zeta),
$$

also for $p_{\Lambda}(\beta, z)$.

As in $[6,25]$ one sees that $p_{\Lambda}(\beta, z)$ is convex in $z$ and $\beta$, and this remains true in the limit $\Lambda=\mathbb{R}^{v}$. Theorem 3.1,(1) (or, alternatively, (4.7) and convexity in $z$ ) also shows that

$$
p_{\Lambda}\left(\beta, z_{1}+z_{2}\right) \geq p_{\Lambda}\left(\beta, z_{1}\right)+p_{\Lambda}\left(\beta, z_{2}\right) .
$$

It is easy to see that

$$
\left\langle F\left(\beta^{1 / 2} \phi\right)\right\rangle_{V}=\langle F(\phi)\rangle_{\beta V},
$$

where $\langle-\rangle_{V}$ is the expectation with respect to the Gaussian measure $d \mu_{V}$ introduced in Section 2.1. (It suffices to prove (4.9) for $F(\phi)=e^{i \phi(g)}, g \in \mathscr{S}$, for which it is obvious).

Equations (4.9), (4.4) and Theorem 3.1,(2) show that

$p_{\Lambda}(\beta, z)$ is monotone increasing in $\beta$;

(this and convexity show that $p_{\Lambda}(\beta, z)$ is also convex in $\beta^{-1}=T$ ).

This completes the proof of Theorem $\mathrm{C},(1)$.

Proof of Theorem $C,(2)$. The proof is given in four steps.

(1) Express the correlation functions $\rho_{\Lambda}\left(\beta, z ;(q)_{N},(x)_{N}\right)$ in terms of the functional integrals given in formula (2.12) of Section 2.1.

(2) In order to apply the inequalities of Theorem 3.1,(5) and Corollary 3.2,(1) we must choose $X=Q \times \mathbb{R}^{v}$ and

$$
d \rho=z g_{\Lambda}^{\beta}(q, x) d \lambda(q) d^{v} x .
$$

We set $C_{1}=C_{2}=V, d \rho_{1 / 2}=z g_{\Lambda_{1 / 2}}^{\beta}(q, x) d \lambda(q) d^{v} x$. If $\Lambda_{1} \subseteq \Lambda_{2}$ then $\rho_{1} \leq \rho_{2}$.

(3) Applying now Corollary 3.2,(1), resp. (3.15), we see that $\rho_{\Lambda}\left(\beta, z ;(q)_{N},(x)_{N}\right)$ is monotone increasing in $\Lambda$ (and also in $z$ ).

(4) Convergence, as $\Lambda \uparrow \mathbb{R}^{v}$, now follows from the uniform bounds of Theorem 2.1, (2), Section 2.2.

By now standard arguments of [14] show that the limiting correlation functions have the same symmetry properties as the potential $V$.

Q.E.D.

Remark. The kind of reasoning employed in the proof of Theorem C, (2) has been introduced in the context of Euclidean field theory in $[23,14]$ and applied in a context similar to the above one in [6]. Presumably it goes back to Griffiths.

Proof of Theorem $Q M$. To prove that the thermodynamic limit of the pressure exists and has the standard properties we repeat the arguments given in the proof 
of Theorem C, (1), but we now define

$$
X=\Omega \times Q \times \mathbb{R}^{v},
$$

where $\Omega$ is the (Wiener) path space. We set

$$
\begin{aligned}
& g_{\Lambda}^{\beta}(\omega, q, x) \equiv e^{(1 / 2) \int_{0}^{\beta} V(q, \omega(\tau) ; q, \omega(\tau)) d \tau} \chi_{\Lambda}(x) \\
& d \rho \equiv d \rho_{\Lambda} \equiv z g_{\Lambda}^{\beta}(\omega, q, x) \operatorname{Pr}_{\Lambda}^{\beta}(q, x, x ; d \omega) d \lambda(q) d^{v} x, z \geq 0 .
\end{aligned}
$$

Define

$$
\Omega_{\Lambda}=\{\omega \in \Omega: \omega(\tau) \in \Lambda, \tau \in[0, \beta]\} .
$$

Then

$$
\operatorname{Pr}_{\Lambda}^{\beta}(q, x, x ; d \omega)=\chi_{\Omega_{A}}(\omega) \operatorname{Pr}^{\beta}(q, x, x ; d \omega),
$$

with $\operatorname{Pr}^{\beta}=\operatorname{Pr}_{\Lambda=\mathbb{R}^{v}}^{\beta} ;$ see $[22,12]$.

From (4.10) we see that $\operatorname{Pr}_{\Lambda}^{\beta}(q, x, x ; d \omega)$ is monotone increasing in $\Lambda$, for all $q$ and $x$. Moreover, for $\Lambda_{1}$ and $\Lambda_{2}$ open sets whose closures are disjoint

$$
\chi_{\Omega_{\Lambda_{1} \cup \Lambda_{2}}}=\chi_{\Omega_{\Lambda_{1}}}+\chi_{\Omega_{\Lambda_{2}}}
$$

on the support of $\operatorname{Pr}^{\beta}(q, x, x ; d \omega)$, a well known decoupling property of Dirichlet boundary conditions.

From (4.10) and (4.11) we get

Lemma 4.1. (1) For $\Lambda_{1}, \Lambda_{2}$ as in (4.11),

$$
d \rho_{\Lambda_{1} \cup \Lambda_{2}}=d \rho_{\Lambda_{1}}+d \rho_{\Lambda_{2}} .
$$

(2) For $\Lambda_{1} \subseteq \Lambda_{2}$

$$
d \rho_{\Lambda_{1}} \leq d \rho_{\Lambda_{2}} \text {. }
$$

(Note that, in the present context, the distribution $l_{x}$ introduced in (3.1) is defined to be

$$
\left.\delta_{q} \delta_{\omega(\tau)} \chi_{[0, \beta]}(\tau)\right) \text {. }
$$

Given Lemma 4.1 and (4.11), the rest of the proof of Theorem QM is essentially identical to the one of Theorem $\mathrm{C}$, (with the exception that the discussion of the properties of $p(\beta, z)$ as a function of $\beta$ is different ; see $[25,21])$.

Q.E.D.

For more details concerning the quantum case see Appendix 1, and [21, 3, 9].

\subsection{Symmetry - and Cluster Properties of Correlation Functions}

Let $\langle\longrightarrow\rangle_{1}(\beta, z)$ denote the equilibrium expectation of the classical, resp. the quantum mechanical systems considered in Theorems $C$ and QM confined to a region $\Lambda$, at inverse temperature $\beta$ and activity $z$. In the classical case,

$$
\rho_{\Lambda}\left(\beta, z ;(q)_{N},(x)_{N}\right)=\left\langle\prod_{j=1}^{N} z: e^{i \beta^{1 / 2} \phi\left(q_{j}, x_{j}\right)}:\right\rangle_{A}(\beta, z) ;
$$

see Equation (2.12), and a similar identity holds in the quantum case: Equation 
(2.17). (The expectation $\langle\longrightarrow\rangle_{A}(\beta, z)$ is given by a probability measure on the dual $\mathscr{S}^{\prime}$ of a nuclear space $\mathscr{S}$; see Section 2).

Proposition 4.2. For all $f \in \mathscr{S}$,

$$
\lim _{\Lambda \uparrow \mathbb{R}^{\nu}}\left\langle e^{i \phi(f)}\right\rangle_{\Lambda}(\beta, z) \equiv\left\langle e^{i \phi(f)}\right\rangle(\beta, z)
$$

exists. The limit is the characteristic functional of a probability measure on $\mathscr{S}^{\prime}$, so that $\langle F\rangle(\beta, z)$ is defined for any measurable function $F$ on $\mathscr{S}^{\prime}$.

Proof. Clearly $\left|\left\langle e^{i \phi(f)}\right\rangle_{\Lambda}(\beta, z)\right| \leq 1$.

Using Theorem 3.1, (5), resp. (3.15) one shows as in the proof of Theorem C (QM), (2) that

$$
\left\langle e^{i \phi(f)}\right\rangle_{\Lambda}(\beta, z)
$$

is monotone increasing in $\Lambda$. Hence the limit exists and is shape independent. Moreover it is obviously a functional of positive type on $\mathscr{S}$, and $\left\langle e^{i \phi(0)}\right\rangle(\beta, z)=$ $\lim \left\langle e^{i \phi(0)}\right\rangle_{\Lambda}(\beta, z)=1$.

Finally

$$
\left\langle\phi(f)^{2}\right\rangle_{\Lambda}(\beta, z) \leq\left\{\begin{array}{l}
(f, V f), \text { in case C } \\
(f, \mathbb{V} f), \text { in case QM, }
\end{array}\right.
$$

uniformly in $\Lambda$. This is the inequality of Corollary 3.2, (2). Hence, $\left\langle e^{i \phi(f)}\right\rangle(\beta, z)$ is continuous in $f$ on $\mathscr{S}$, (a standard argument). The proof is completed by applying Minlos' theorem.

By (4.12) and Proposition 4.2 the classical correlation functions in the thermodynamic limit are given by

$$
\rho\left(\beta, z ;(q)_{N},(x)_{N}\right)=\left\langle\prod_{j=1}^{N} z: e^{i \beta^{1 / 2} \phi\left(q_{j}, x_{j}\right)}:\right\rangle(\beta, z)
$$

and the quantum mechanical density correlation functions by

$$
\rho\left(\beta, z ;(q)_{N},(x)_{N}\right)=\left\langle\prod_{j=1}^{N} z \int \operatorname{Pr}^{\beta}\left(q_{j}, x_{j}, x_{j} ; d \omega_{j}\right): e^{i \int_{0}^{\beta} d \tau \phi\left(q_{j}, \omega_{j}(\tau), \tau\right)}:\right\rangle(\beta, z) .
$$

See (2.17). Let $\xi \equiv x$, in the classical case, and $\xi \equiv(x, \tau)$, in the quantum mechanical case. The expectation

$$
\left\langle\phi(q, \xi) \phi\left(q^{\prime}, \xi^{\prime}\right)\right\rangle(\beta, z)
$$

is called "effective potential function".

We define a susceptibility $\chi$ by

$$
\chi(q, \beta, z) \equiv \lim _{B \rightarrow \infty}|B|^{-1} \int_{B \times B} d \xi d \xi^{\prime}\left\langle\phi(q, \xi) \phi\left(q, \xi^{\prime}\right)\right\rangle(\beta, z) .
$$

Theorem 4.3. Under the hypotheses of Theorems C, resp. $Q M$ the following is true: (1) The expectation $\langle-\rangle(\beta, z)$ (in particular, all correlation functions in the thermodynamic limit) has all the symmetry properties of the potential $V$ : If $V$ is translation- 
invariant, rotation-invariant, (resp.-covariant, ... ) then so is $\langle-\rangle(\beta, z)$. The correlation functions are monotone increasing in the activity $z$ and in the measure $d \lambda$.

(2) The effective potential function $\langle\phi(\bar{f}) \phi(f)\rangle(\beta, z)$ is monotone decreasing in $z$ and $\lambda$, and

$$
0 \leq\langle\phi(\bar{f}) \phi(f)\rangle(\beta, z) \leq\left\{\begin{array}{l}
(f, V f) \text { in case } C \\
(f, \mathbb{V} f) \text { in case } Q M
\end{array}\right.
$$

for arbitrary complex-valued $f$. In particular

$$
\chi(q, \beta, z) \leq \chi(q, \beta, 0) .
$$

Proof. The first part of (1) follows from monotonicity of $\left\langle e^{i \phi(f)}\right\rangle_{\Lambda}(\beta, z)$ in $\Lambda$, as already noted in the proof of Theorem $C,(2)$ : see [14]. The second part of (1) follows from Corollary 3.2,(1) and (3.15). Finally, (2) is Corollary 3.2, (2).

Q.E.D.

Remark. The effective potential function (resp. the susceptibility) is often a reliable measure for the rate of decay of arbitrary correlations (see e.g. Theorem 4.4 below). In such a case the upper bound of Theorem 4.3 can often be used to show that there is no long range order ("absence of phase transitions").

Next we consider a classical, neutral two-component gas such as introduced in Section 1, Example (A):

$$
\begin{aligned}
V\left(q, x ; q^{\prime}, x^{\prime}\right) & =q \cdot q^{\prime}(2 \pi)^{-v / 2} \int e^{i k\left(x-x^{\prime}\right)} \hat{V}(k) d^{v} k, \\
\text { with } \quad 0 & \leq \hat{V}(k) \in L^{1}\left(\mathbb{R}^{v}\right) .
\end{aligned}
$$

We define $\Gamma(x)=-(2 \pi)^{-v / 2} \int e^{i k x} \hat{V}(k)^{-1} d^{v} k$, (in the distribution sense).

Theorem 4.4. Suppose that $\Gamma(x) \geq 0$, for $x \neq 0$ (in the distribution sense).

Then the expectation $\langle->(\beta, z)$ is clustering. In particular,

$$
\begin{aligned}
& \lim _{|a| \rightarrow \infty} \rho\left(\beta, z ;(q)_{N}, x_{1}, \ldots, x_{M}, x_{M+1}+a, \ldots, x_{N}+a\right) \\
& \stackrel{=}{=} \rho\left(\beta, z ;\left(q_{1}, x_{1}\right), \ldots,\left(q_{M}, x_{M}\right)\right) \rho\left(\beta, z ;\left(q_{M+1}, x_{M+1}\right), \ldots,\left(q_{N}, x_{N}\right)\right) .
\end{aligned}
$$

Proof. There is a minor cheat in the statement of Theorem 4.4: What we really need is that $\langle-\rangle(\beta, z)$ satisfies the $F K G$ correlation inequalities [5, 14]. These inequalities are usually proven for classical lattice systems. In the case of a classical, neutral two-component lattice gas (with configuration space $\varepsilon \mathbb{Z}^{v}, \varepsilon>0$, rather than $\left.\mathbb{R}^{v}\right)$ the hypotheses of Theorem 4.4 guarantee that $\langle-\rangle(\beta, z)$ satisfies the FKG inequalities [14].

Next, one must show that the lattice expectations converge, as $\varepsilon \downarrow 0,[14]$. For a large class of potentials $V$ this "convergence of the lattice approximation" can be checked; see e.g. $[6,8]$. The formal limiting condition on $V$ guaranteeing that $\langle-\rangle(\beta, z)$ satisfies the FKG inequalities is then just $\Gamma(x) \geq 0$, for $x \neq 0$. Once the FKG inequalities are proven for $\langle-\rangle(\beta, z)$, a theorem due to Lebowitz [17] and Simon [27] says that the expectation $\langle-\rangle(\beta, z)$ clusters if and only if the effective potential function clusters. So we are left with showing

$$
\langle\phi(0) \phi(x)\rangle(\beta, z) \rightarrow 0 \text {, as }|x| \rightarrow \infty \text {. }
$$


By Theorem 4.3, (2)

$$
0 \leq\left\langle|\phi(f)|^{2}\right\rangle(\beta, z) \leq \int d^{v} k|\hat{f}(k)|^{2} \hat{V}(k),
$$

for all $f$ with $\hat{f} \in L^{\infty}\left(\mathbb{R}^{v}\right)$. Hence

$$
0 \leq\langle\hat{\phi}(k) \hat{\phi}(-k)\rangle(\beta, z) \leq \hat{V}(k),
$$

in the sense of measurable functions. Together with (4.17) this implies that

$$
\langle\hat{\phi}(k) \hat{\phi}(-k)\rangle(\beta, z) \in L^{1}\left(\mathbb{R}^{v}\right) \text {. }
$$

By the Riemann-Lebesgue lemma,

$$
\langle\phi(0) \phi(x)\rangle(\beta, z)=(2 \pi)^{-v / 2} \int e^{-i k x}\langle\hat{\phi}(k) \hat{\phi}(-k)\rangle(\beta, z) d^{v} k
$$

tends to 0 , as $|x| \rightarrow \infty$.

Q.E.D.

Remarks. 1. Theorem 4.4 proves that in the sense that the expectation $\langle\longrightarrow\rangle(\beta, z)$ is clustering (and hence is ergodic under translations) there are no phase transitions in those gases that satisfy the hypotheses of Theorem 4.4. We conjecture that, for these gases, $\langle-\rangle(\beta, z)$ is the only Gibbs equilibrium expectation in the thermodynamic limit. A partial result in this direction may follow by adapting the methods of $[19,20,11]$, certainly for the lattice gases. Our conjecture ought to be true for all the classical gases with two body potentials of positive type converging to 0 , as $\left|x-x^{\prime}\right| \rightarrow \infty$ which satisfy the neutrality condition (1.4).

2. Theorem 4.4 applies to the classical, neutral two-component Yukawa gas in two dimensions studied in [6], for all $\beta$ and $z$, besides a large class of lattice gases, including lattice Coulomb gases in $v \geq 3$ dimensions [7], already mentioned in the proof. These lattice gases are of considerable interest for the theory of higher order phase transitions and gauge quantum field theories; see $[7,9]$.

Next, suppose that the potential $V$ has the property that

$$
V(q, x ; q, x) \equiv v(q)
$$

is independent of $x$.

We then define

$$
d \lambda_{\beta}(q) \equiv e^{-(\beta / 2) v(q)} d \lambda(q) \text {. }
$$

Theorem 4.5. In addition to the hypotheses of Theorem $C$, resp. $Q M$ assume that, in the definition of $\langle-\rangle(\beta, z)$, the measure $d \lambda$ is replaced by $d \lambda_{\beta}$. (This amounts to undoing Wick order). Then $\beta\left\langle|\phi(f)|^{2}\right\rangle(\beta, z)$ is monotone increasing in $\beta$. In particular

$$
\beta\langle\hat{\phi}(q, k) \hat{\phi}(q,-k)>(\beta, z) \text { and } \beta \chi(q, \beta, z)
$$

are monotone increasing in $\beta$.

Proof. This follows immediately from identity (4.9) and Corollary 3.2, (2).

Remarks. The monotonicity properties of $\beta \chi(q, \beta, z)$ in $\beta$ and $z$ (see Theorem 4.3) are useful in the theory of higher order phase transitions, in particular in the 
discussion of Debye screening [2] and its breakdown [7, 9] in Coulomb-type lattice gases.

The following result, valid under the hypotheses of Theorem C, resp. QM, is an inequality for an Ursell (connected) function "inverse" to the well known Lebowitz inequality for Ising models [18].

Theorem 4.6. ("Inverse" Lebowitz Inequality). Let $h\left((q)_{4} ;(x)_{4}\right)$ be some measurable function on $\left(Q \times \mathbb{R}^{v}\right)^{\times 4}$ with the property that $(h, f \otimes f \otimes f \otimes f) \geq 0$, for all real, measurable functions $f$ on $Q \times \mathbb{R}^{v}$. Then

$$
\int \prod_{i=1}^{4} d \lambda\left(q_{i}\right) d^{v} x_{i} h\left((q)_{4} ;(x)_{4}\right)\left\langle\prod_{j=1}^{4} \phi\left(q_{j}, x_{j}\right)\right\rangle^{C}(\beta, z) \geq 0,
$$

where $\langle-\rangle^{C}(\beta, z)$ denotes the connected (truncated) expectation.

The proof of this theorem is sketched in Appendix 2. Although this inequality can be used, in both case $C$ and case QM, to derive upper bounds on $(\partial / \partial \beta)[\beta \chi(q$, $\beta, z)]^{-1}$ and other quantities involving the effective potential function, it might not be much more than a curiosity. Some applications of it are made in [9]. (Note that first order perturbation theory in $z$ predicts Theorem 4.6).

Remarks. In the case of lattice gases $\left(\mathbb{R}^{v}\right.$ replaced by $\left.\mathbb{Z}^{v}\right)$ the limit $z \rightarrow+\infty$ is of considerable interest, as it yields important, classical lattice spin systems. As an example we mention that the $z=+\infty$ limit of the lattice Coulomb gas is the dual Villain ("integer bed spring") model which in two dimensions is closely related to the classical rotator model [16] and the abelian Higgs lattice gauge theory [7]. For these limiting systems all the correlation inequalities of Sections III and IV and their consequences hold and provide information about the thermodynamic limit and the critical behaviour of such lattice spin systems. In this connection it is of interest to note that exponential Debye screening in the lattice Coulomb gas breaks down, and $\chi(\beta, z)$ is infinite, for all those $\beta$ for which the limiting dual Villain model has infinite susceptibility: A consequence of Theorem 4.3, (2). See also [9].

\section{Generalizations and Comments}

In this section we sketch generalizations of the results of Sections $1-4$. We content ourselves with giving the main ideas only, omitting full details.

First, we consider a class of two body potentials of positive type satisfying a Dirichlet type decoupling property and we add a positive potential to them. We are interested in constructing the thermodynamic limit for the pressure. Let $V\left(q, x ; q^{\prime}, x^{\prime}\right)$ be a translation invariant two body potential of positive type. Let $\Lambda$ be an open region in $\mathbb{R}^{v}$. We assume that we can impose Dirichlet type boundary conditions on $V: V \rightarrow V_{A}$, with the property that $V_{A}$ still has all the properties of $V$ stated in Section 1 (positive type, neutrality condition (1.4), etc.) and, in addition,

$$
V_{\Lambda}\left(q, x ; q^{\prime}, x^{\prime}\right)=0
$$

if $x$ and $x^{\prime}$ are separated by $\partial \Lambda$; and

$$
\left.V_{\Lambda}^{-1} \geq V_{\tilde{\Lambda}}^{-1} \quad \text { (i.e. } V_{\tilde{\Lambda}} \geq V_{\Lambda}\right)
$$

if $\partial \Lambda \supset \partial \tilde{\Lambda}$, (in the sense of quadratic forms). Let $V_{+}\left(x-x^{\prime}\right)$ be an additional, trans- 
lation invariant, positive potential (e.g. a hard core type potential), and set

$$
V_{\Lambda}^{\text {tot, }}=V_{\Lambda}+V_{+} \text {. }
$$

Let $\Xi_{A}(\beta, z)$ denote the partition function of the (classical or quantum mechanical) system of distinguishable particles confined to the region $\Lambda$, interacting via two body potential $V_{A}^{\text {tot, }}$ and with a self interaction $\frac{1}{2}\left(V-V_{A}\right)\left(q_{j}, x_{j} ; q_{j}, x_{j}\right)$. Finally $p_{A}(\beta, z)$ is the pressure of this system.

Theorem 5.1. If $\Lambda \rightarrow \mathbb{R}^{v}$, in the sense of van Hove,

$$
\lim _{\Lambda \rightarrow \mathbb{R}^{v}} p_{\Lambda}(\beta, z) \equiv p(\beta, z)
$$

exists.

Remarks. Again no assumption on the range of $V$ and $V_{+}$have to be made to prove the existence of the limit of $p_{A}(\beta, z)$, as $\Lambda \uparrow \mathbb{R}^{v}$. However, in order to show that $p(\beta, z)=\lim _{\Lambda \uparrow \mathbb{R}^{v}} p_{\Lambda}(\beta, z)>0$ (i.e. $p(\beta, z)$ is non-trival) an additional argument, not discussed here, is required. This yields restrictions on the range of $V_{+}$, e.g. tempering, [25]. Theorem 5.1 applies e.g. to the classical, two component Coulomb gas in any number of dimensions, provided the particles have a hard core which makes the system stable. (For the case of two dimensions see [6]). Theorem 5.1 is of some interest, because it gives a construction of the thermodynamic limit of the pressure even for systems with long range interactions that include positive potentials. Such systems are expected to have phase transitions (formation of crystals, etc.) which do not seem to occur in the systems considered in Sections 1-4, (see Theorem 4.4!). Another (possibly more satisfactory) version of Theorem 5.1 appears in [9], where no use of Dirichlet boundary conditions is made.

Proof. First we recall the estimate

$$
1 \leq \Xi_{\Lambda}(\beta, z) \leq e^{\zeta K_{\beta}|\Lambda|},
$$

where $z$ and $\zeta$ are positive, and $K_{\beta}$ is independent of $\Lambda$. The lower bound is trivial, the upper bound is proven as follows: Clearly $\Xi_{A}(\beta, z)$ increases if we set $V_{+} \equiv 0$. When $V_{+} \equiv 0$ (5.4) is Theorem 2.1, (1) which is still true in this case, because we have assumed $V_{A}$ to share all properties of $V$. Some standard arguments that we do not reproduce here (see $[25,21,15])$ show that estimate (5.4) reduces the proof of Theorem 5.1 to the following problem: Let $\Lambda$ be an open cube and $\Lambda_{n} \equiv\{x$ : $\left.\frac{1}{n} x \in \Lambda\right\}, n=2,3, \ldots$. Show that

$$
p_{A_{n}}(\beta, z) \text { converges, as } n \rightarrow \infty \text {. }
$$

To prove this, we cover $\Lambda_{n}$ with $n^{v}$ disjoint copies of $\Lambda$. The resulting open set is denoted $\tilde{\Lambda}_{n}$. With $\tilde{\Lambda}_{n}$ we associate the partition function $\Xi_{\tilde{A}_{n}}(\beta, z)$ of the system in $\tilde{\Lambda}_{n}$ which has the property that particles located in different copies of $\Lambda$ do not interact with each other. This means that

$$
\Xi_{\tilde{A}_{n}}(\beta, z)=\Xi_{\Lambda}(\beta, z)^{n v} .
$$


Let $U_{\tilde{\Lambda}_{n}}^{+}\left(m_{1}, \ldots, m_{n^{v}}\right)$ denote the potential of $\sum_{j=1}^{n^{v}} m_{j}$ particles with two body potential $V_{+}, m_{j}$ of which are located in the $j^{\text {th }}$ copy $\Lambda_{j}$ of the cube $\Lambda$, subject to the restriction that all interactions between particles in different copies of $\Lambda$ are omitted; the total potential without this restriction is denoted $U^{+}\left(m_{1}, \ldots, m_{n v}\right)$.

Since $V_{+}$is positive, we have

$e^{-U^{+}\left(m_{1}, \ldots, m_{n} v\right)} \leq e^{-U \stackrel{+}{\tilde{\Lambda}}_{n}\left(m_{1}, \ldots, m_{n} v\right)}$,

pointwise.

Furthermore,

$$
\begin{aligned}
& \left\langle\prod_{j=1}^{n^{v}} \prod_{l_{j}=1}^{m_{j}} e^{(\beta / 2) V\left(q_{l_{j}}, 0 ; q_{l_{j}}, 0\right)} \cos \phi\left(q_{l_{j}}, x_{l_{j}}\right)\right\rangle_{V_{\Lambda_{n}}} \\
& \leq\left\langle\prod_{j=1}^{n^{v}} \prod_{l_{j}=1}^{m_{j}} e^{(\beta / 2) V\left(q_{l_{j}}, 0 ; q_{l_{j}}, 0\right)} \cos \phi\left(q_{l_{j}}, x_{l_{j}}\right)\right\rangle_{V \tilde{\Lambda}_{n}},
\end{aligned}
$$

by Corollary 3.2, (1).

Hence

$$
\Xi_{A_{n}}(\beta, z) \leq \Xi_{\tilde{\Lambda}_{n}}(\beta, z),
$$

by (5.7), (2.3) and (5.8). Combination of (5.6) and (5.9) implies that $p_{\Lambda_{n}}(\beta, z)$ is monotone decreasing in $n$, i.e. we have proven (5.5).

Q.E.D.

Our last generalization concerns extending our construction of the thermodynamic limit of the pressure to classical or quantum mechanical systems of distinguishable particles interacting via translation invariant two body potentials of positive type with logarithmic singularities at 0 distance. For simplicity we only consider the case

$$
V\left(q, x ; q^{\prime}, x^{\prime}\right)=q \cdot q^{\prime} V\left(x-x^{\prime}\right),
$$

where $q^{\left({ }^{\prime}\right)}= \pm 1$

$$
|V(x)| \leq \text { const. } \log \left[\frac{1}{|x|}+2\right]
$$

and

$$
V \in L^{1}\left(\mathbb{R}^{v}, d^{v} x\right) .
$$

For such potentials we prove some sort of stability (see [6]) at high enough temperature.

Theorem 5.2. For the systems introduced above the thermodynamic limit of the pressure (with boundary conditions as in Theorem C, resp. QM) exists and is finite for all $\beta<\beta_{0}(V)$, where $\beta_{0}(V)$ is some strictly positive, but generally finite constant.

Remarks. (1) If the upper bound on $V(x)$ assumed in (5.10) is saturated for small $|x|$ then there exists some $\bar{\beta}_{0}(V)$ such that $e^{\beta V(x)}$ is not integrable at $x=0$, for all $\beta \geq \bar{\beta}_{0}(V)$. In this case the system collapses at some temperature $\beta_{0}^{-1} \geq \bar{\beta}_{0}(V)^{-1}$. 
The estimates on $\beta_{0}=\beta_{0}(V)$ obtained in the proof of Theorem 5.3 are rather crude and give $\beta_{0}(V)<\bar{\beta}_{0}(V)$, whenever $v>2$.

(2) We have also proven that the "correlations" $\left\langle\prod \phi\left(q_{i}, x_{i}\right)\right\rangle_{\Lambda}(\beta, z)$ (in the notation of (4.12) and Proposition 4.2) converge as $\Lambda \uparrow \mathbb{R}^{v}$. This follows from Theorem 5.2 and the observation that $\left\langle e^{\phi(f)}\right\rangle_{\Lambda^{(\beta, z)}}$ is monotone decreasing in $\Lambda$ (another simple correlation inequality) and bounded for $\Lambda=\emptyset$, for all $\mathrm{f} \in \mathscr{S}$. For the standard correlation functions an analogous result requires a proof of uniform upper bounds which is non-trivial and not attempted here.

Proof. Using Theorem 3.1,(1) we see that Theorem 5.2 is true, once we have proven stability in the form of an exponential upper bound on the partition function for small enough $\beta$; see [6]. The Golden-Thompson inequality shows that it suffices to prove stability in the classical case. (This also implies stability for the corresponding quantum system with Fermi statistics, for arbitrary $\beta,[6])$.

The basic idea is now to reduce the proof of stability to the one for the two dimensional, two component, classical Yukawa gas given in [6]. Simple considerations show that, given a potential $\mathrm{V}$ satisfying $(5.10)-(5.11)$, there exists a potential $W$ of finite range $R$ with the properties

(1) $W$ is of positive type,

(2) $\hat{W}(k) \geq \hat{V}(k)$, for all $k \in \mathbb{R}^{v}$; (i.e. $W \geq V$ in the sense of quadratic forms),

(3) $|W(x)| \leq$ const $^{\prime} \log \left(\frac{1}{|x|}+2\right)$.

Let $\Xi_{\Lambda}^{V}(\beta, z), \Xi_{\Lambda}^{W}(\beta, z)$ denote the grand canonical partition functions for potentials $V, W$, respectively.

If we apply Wick ordering (see Eq. (2.3)) on both sides of the conditioning inequality (Theorem 3.1, (2)) we obtain

$$
\Xi_{\Lambda}^{V}(\beta, z) \leq \Xi_{\Lambda}^{W}(\beta, z)
$$

see [6] for a detailed discussion of such inequalities. Hence it suffices to prove stability for $\Xi_{A}^{W}(\beta, z)$. By monotonicity of $\Xi_{\Lambda}^{W}(\beta, z)$ in $\Lambda$, proven in Theorem 3.1,(1) we may assume $\Lambda$ to be a union of cubes $\Delta_{j}$ with sides of length $4 \mathrm{R}$. We divide each cube $\Delta_{j}$ of this cover of $\Lambda$ into $2^{v}$ disjoint cubes $\Delta_{j}^{l}, l=1, \ldots, 2^{v}$ with sides of length $2 \mathrm{R}$; clearly dist $\left(\Delta_{j}^{l}, \Delta_{j^{\prime}}^{l}\right) \geq 2 \mathrm{R}$, for $j \neq j^{\prime}$, (independently of $l$ ). By Equation (2.11) of Section 2 we have

$$
\Xi_{\Lambda}^{W}(\beta, z)=\left\langle e^{z C_{A}^{\beta}}\right\rangle_{W},
$$

and, by definition (2.9) of $C_{\Lambda}^{\beta}$,

$$
C_{A}^{\beta}=\sum_{l=1}^{2 v} \sum_{j} C_{\Delta_{j}^{\prime}}^{\beta} \text {. }
$$

We now apply the Hölder inequality with respect to the Gaussian expectation $\langle-\rangle_{W}$ to separate adjacent cubes:

$$
\Xi_{\Lambda}^{W}(\beta, z)=\left\langle e^{z \sum_{l=1}^{2 v} \sum_{j} C_{d_{j}^{j}}^{\beta_{j}}}\right\rangle_{W}
$$




$$
\begin{aligned}
& =\left\langle\prod_{l=1}^{2 v} e^{z \sum_{J} C_{\Delta_{j}^{j}}^{\beta}}\right\rangle_{W} \\
& \leq \prod_{l=1}^{2 v}\left\langle e^{2 v_{z} \sum_{j} C_{\Delta_{j}^{\prime}}^{\beta}}\right\rangle_{W}^{2^{-v}} .
\end{aligned}
$$

(The idea of separating adjacent cubes and using inequality (5.15) in the proof of stability is taken from [6]). Next we recall that

$$
\operatorname{dist}\left(\Delta_{j^{\prime}}^{l}, \Delta_{j^{\prime}}^{l}\right) \geq 2 R,
$$

for all $l$ and $j \neq j^{\prime}$, but that the range of $W$ is $R$. Hence

$$
\begin{aligned}
\left\langle e^{2 v_{z} \sum_{j} C_{\Delta_{j}^{j}}^{\beta}}\right\rangle_{W} & =\left\langle\prod_{j} e^{2^{2} C_{z} C_{\Delta_{j}^{\prime}}^{\beta}}\right\rangle_{W}, \\
& =\prod_{j}\left\langle e^{2 v_{z} C_{\Delta_{j}^{j}}^{\beta}}\right\rangle_{W},
\end{aligned}
$$

by inspection.

By translation invariance the factors on the r.h.s. of (5.16) are independent of $j$ and $l$. Thus stability is proven if we can show that $\left\langle e^{2^{v_{z}} C_{\Delta}^{\beta_{\Delta}}}\right\rangle_{W}$ is finite, where $\Delta \stackrel{\text { e.g. }}{=} \Delta{ }_{1}^{1}$.

For $v=2$ this follows from [6], for small enough $\beta$, (temperature above collapse temperature). The same methods that were developed in [6] for $v=2$ also work for $v=1$. For $v \geq 3$, we note that

$$
\begin{aligned}
& e^{2 v_{z} \int_{\Delta} d x_{1} d x_{2}} \prod_{\alpha=3}^{v} d x_{\alpha}: \cos \beta^{1 / 2} \phi(x): \\
& \leq(2 R)^{-v+2} \int_{0 \leq x_{\alpha} \leq 2 R} \prod_{\alpha=3}^{v} d x_{\alpha} e^{2^{v_{z}(2 R)^{\nu-2}} \int_{0 \leq x_{1 / 2} \leq 2 R} d x_{1} d x_{2} \cdot \cos \beta^{1 / 2} \phi(x):},
\end{aligned}
$$

by Jensen's inequality. Using the linearity of $\langle-\rangle_{W}$ and translation invariance we conclude

$$
\left\langle e^{2^{v} z C_{A}^{\beta}}\right\rangle_{W} \leq\left\langle e^{2^{v_{z}(2 R)^{v-2}} \int_{0 \leq x_{1 / 2} \leq 2 R} d x_{1} d x_{2}: \cos \beta^{1 / 2} \phi(x):}\right\rangle_{W} .
$$

By applying conditioning (Theorem 3.1, (2)) once more the r.h.s. can be dominated by the partition function of a two dimensional Yukawa gas in a square with sides of length $2 \mathrm{R}$, at activity $z^{\prime}=2^{v} z(2 R)^{v-2}$ and inverse temperature $\beta^{\prime}=C \beta$, where $C \geq 1$ is some finite constant. The latter has been shown to be finite for $\left(\beta^{\prime}\right)^{2}<4 \pi$; see [6]. This proves stability for $\beta^{2}<4 \pi C^{-2}\left(\leq \beta_{0}(V)^{2}\right)$.

Q.E.D.

Remarks. As noted, the estimates used in the proof of Theorem 5.2 are rather crude, in particular (5.17) is a bad estimate. It yields stability only when $\beta$ is such that

$$
e^{\beta V(x)}<0\left(|x|^{-2}\right), \text { for }|x| \ll 1,
$$


whereas the correct condition should probably be

$$
e^{\beta V(x)}<0\left(|x|^{-v}\right) \text {, for }|x| \ll 1 \text {, }
$$

as is the case for $v=1,2$.

(2) Uniform estimates on the correlation functions have been proven in $[6,11]$ for $v=2$, and the techniques developed there also work for $v=1$. For $v \geq 3$ new methods are needed. It would suffice to show that $\langle\longrightarrow\rangle_{A}(\beta, z)$ is locally $L^{p}$ with respect to $\langle-\rangle_{W}$, with an $L^{p}$ norm bounded uniformly in $\Lambda$. For $v=1,2$ this can be shown using a "transfer matrix", [11]. For $v \geq 3$ such transfer matrices do not exist.

Note Added. After completion of this paper we learned of work by A. J. F. Siegert, Ref. [29], which contains most of Section 2. We thank A. Lenard for informing us of Ref. [29]. We also thank E. B. Davies for several helpful comments.

\section{Appendix 1}

In this appendix we indicate briefly how to construct imaginary time Green's functions (ITGF's) for the gases of distinguishable, quantum mechanical particles considered in this paper. The hypotheses of Theorem QM are adopted throughout this appendix.

In a system of infinitely many particles we single out $N$ specific particles. A "configuration" $c_{j}$ of the $j^{\text {th }}$ particle is a collection $\left\{x_{l}^{j}, \tau_{l}^{j}\right\}_{l=1}^{L_{j}}$ of space points $x_{l}^{j}$ and imaginary times $\tau_{l}^{j}$ with $0 \leq \tau_{1}^{j}<\ldots<\tau_{L_{j}}^{j}<\beta$.

We propose to construct the joint probability

$$
\rho\left(\beta, z ; c_{1}, \ldots, c_{N}\right)
$$

of the configurations $c_{1}, \ldots, c_{N}$ of $N$ particles with generalized charges $q_{1}, \ldots, q_{N}$ in the Gibbs state at inverse temperature $\beta$ and activity $z$, in the thermodynamic limit, defined by the condition that the imaginary time path $\omega_{j}$ of the $j^{\text {th }}$ particle obeys the restrictions

$$
\omega_{j}\left(\tau_{l}^{j}\right)=x_{l}^{j}, l=1, \ldots, L_{j},
$$

for all $j=1, \ldots, N$.

First we derive an expression for this probability in the case where the system is confined to a bounded, open region $\Lambda \subset \mathbb{R}^{v}$. It has an obvious definition in terms of the kernel

$$
P_{\Lambda}^{\tau}\left((q)_{N} ;(x)_{N},\left(x^{\prime}\right)_{N}\right)
$$

of the Hamiltonian semigroup $\exp \left[-\tau H_{\Lambda}^{N}\left((q)_{N}\right)\right]$ : Let $\left\{s_{\alpha}\right\}_{\alpha=1}^{L}$ be a permutation of the set $\bigcup_{j=1}^{N}\left\{\tau_{l}^{j}\right\}_{l-1}^{L_{j}}$ with the property

$$
0 \leq s_{1}<s_{2}<\ldots<s_{L}<\beta .
$$

Let $\pi_{k}$ be a permutation of $\{1, \ldots, N\}$ such that

$$
\tau_{l_{1}}^{\pi_{k}(1)}=\ldots=\tau_{l_{N_{k}}}^{\pi_{k}\left(N_{k}\right)}=s_{k},
$$


for some $l_{1}, \ldots, l_{N_{k}}$, and $\tau_{l}^{\pi_{k}(m)} \neq s_{k}$, for all $m>N_{k}$ and all $l=1, \ldots, L_{\pi_{k}(m)}$.

Then

$$
\begin{aligned}
\rho_{\Lambda}\left(\beta, z ; c_{1}, \ldots, c_{N}\right) \equiv & \Xi_{\Lambda}(\beta, z)^{-1} \sum_{M=0}^{\infty} \frac{z^{N+M}}{M !} \\
& \cdot \int_{Q^{M}} d \lambda\left(q^{\prime}\right)_{M} \int \ldots \int_{\Lambda} \prod_{\Lambda=1}^{L}\left\{\prod_{j=N_{k}+1}^{N} d^{v} x_{\pi_{k}(j)}^{k} d\left(y^{k}\right)_{M}\right\} \\
& \cdot \prod_{k=1}^{L-1} P^{s_{k+1}-s_{k}}\left((q)_{N}\left(q^{\prime}\right)_{M} ;\left(x^{k}\right)_{N}\left(y^{k}\right)_{M},\left(x^{k+1}\right)_{N}\left(y^{k+1}\right)_{M}\right) \\
& \cdot P_{\Lambda}^{\beta-s_{L}+s_{1}}\left((q)_{N}\left(q^{\prime}\right)_{M} ;\left(x^{1}\right)_{N}\left(y^{1}\right)_{M},\left(x^{L}\right)_{N}\left(y^{L}\right)_{M}\right) .
\end{aligned}
$$

We propose to reexpress the r.h.s. of (A.1.2) in terms of functional integrals. Given a configuration $c=\left\{x_{l}, \tau_{l}\right\}_{l=1}^{L}$, let $\operatorname{Pr}_{\Lambda}^{\beta}(q, c ; d \omega)$ be the conditional Wiener measure concentrated on all paths $\omega \in \Omega$ with the properties

$$
\begin{aligned}
& \omega(\tau) \in \Lambda, \text { for all } 0 \leq \tau \leq \beta \\
& \omega\left(\tau_{l}\right)=x_{l}, l=1, \ldots, L,
\end{aligned}
$$

determined by the Laplacean with 0 -Dirichlet data at $\partial \Lambda$.

Let $\Sigma_{\left[\tau_{1}, \tau_{2}\right]}$ denote the $\sigma$-algebra on path space $\Omega$ generated by all functions $f\left(\omega\left(s_{1}\right), \ldots, \omega\left(s_{n}\right)\right), f \in C\left(\mathbb{R}^{v n}\right), n=1,2, \ldots$, with $\tau_{1} \leq s_{1}<\ldots<s_{n} \leq \tau_{2}$.

Lemma A.1.1. Let $F_{1}$ be a $\Sigma_{\left[\tau_{1}, \tau_{2}\right]}$-measurable function on $\Omega$ and $F_{2}$ a $\Sigma_{\left[\tau_{2}, \tau_{3}\right]}$ measurable function on $\Omega$.

Then

$$
\begin{aligned}
& \int_{\Lambda} d^{v} x \int_{\Omega} \operatorname{Pr}_{\Lambda}^{\tau_{2}-\tau_{1}}\left(q, x_{1}, x ; d \omega\right) F_{1}(\omega) \int_{\Omega} \operatorname{Pr}_{\Lambda}^{\tau_{3}-\tau_{2}}\left(q, x, x_{2} ; d \omega\right) F_{2}(\omega) \\
& \quad=\int_{\Omega} \operatorname{Pr}_{\Lambda}^{\tau_{3}-\tau_{1}}\left(q, x_{1}, x_{2} ; d \omega\right) F_{1}(\omega) F_{2}(\omega) .
\end{aligned}
$$

Remark. This equation is well known, so that we omit the proof.

From the Feynman-Kac formula (1.18), definition (A.1.2) and repeated application of Lemma A.1.1 we obtain, upon comparison with (2.14)-(2.17).

Theorem A.1.2. $\rho_{\Lambda}\left(\beta, z ; c_{1}, \ldots, c_{N}\right)$

$$
=\Xi_{\Lambda}(\beta, z)^{-1} z^{N}\left\langle\prod_{j=1}^{N} \int_{\Omega} \operatorname{Pr}_{\Lambda}^{\beta}\left(q_{j}, c_{j} ; d \omega_{j}\right): e^{i \int_{0}^{\beta} d \tau \phi\left(q_{J}, \omega_{J}(\tau), \tau\right)}: e^{z \mathbb{C}_{\Lambda}^{\beta}}\right\rangle_{V} .
$$

By construction of the Wiener measure for the Laplacean with O-Dirichlet data at $\partial \Lambda$ it follows immediately that

$\operatorname{Pr}_{\Lambda}^{\beta}(q, c ; d \omega)$

is monotone increasing in $\Lambda$. This permits us to apply the correlation inequalities of Section 3 to show that $\rho_{\Lambda}\left(\beta, z ; c_{1}, \ldots, c_{N}\right)$ is monotone increasing in $\Lambda$.

Assuming that $\sup V(q, x ; q, x)$ is finite, the methods of Section 2 yield uniform 
upper bounds on $\rho_{\Lambda}\left(\beta, z ; c_{1}, \ldots, c_{N}\right)$, and this combined with monotonicity in $\Lambda$ proves the existence of the thermodynamic limit.

Remark. From the collection $\left\{\rho\left(\beta, z ; c_{1}, \ldots, c_{N}\right)\right\}$, for arbitrary $c_{1}, \ldots, c_{N}$ arbitrary $N=0,1,2, \ldots$, one can reconstruct a $\beta-$ KMS state by analytic continuation in the time arguments; [26].

\section{Appendix 2}

Here we prove the "inverse" Lebowitz inequality of Theorem 4.6, i.e.

$$
\int \prod_{i=1}^{4} d \lambda\left(q_{i}\right) d^{v} x_{i} h\left((q)_{4} ;(x)_{4}\right)\left\langle\prod_{j=1}^{4} \phi\left(q_{j}, x_{j}\right)\right\rangle^{c}(\beta, z) \geq 0
$$

for all h, for which

$$
\int \prod_{i=1}^{4} d \lambda\left(q_{i}\right) d^{v} x_{i} h\left((q)_{4} ;(x)_{4}\right) \prod_{i=1}^{4} f\left(q_{i}, x_{i}\right) \geq 0
$$

for arbitrary real functions $f$ on $Q \times \mathbb{R}^{v}$.

Our proof of this inequality is presented within the general framework introduced in Section 3, and the notations used there are kept throughout this appendix. Following [18] and [28] we introduce four independent copies of our system, i.e. we make use of four independent random fields $\phi, \chi, \phi^{\prime}$ and $\chi^{\prime}$ with identical distribution given by the probability measure

$$
d \mu(\phi) \equiv \Xi(C, \rho)^{-1} \exp \left\{\int_{X} d \rho(x) \cos \phi\left(l_{x}\right)\right\} d \mu_{C}(\phi),
$$

where $d \mu_{C}$ is the Gaussian measure with mean 0 and covariance $C$; see Section 3 for definitions.

Next we define four new random fields $\alpha, \beta, \gamma, \sigma$ by the equations

$$
\left.\begin{array}{l}
\alpha=\frac{1}{2}\left(\phi+\chi+\phi^{\prime}+\chi^{\prime}\right), \beta=\frac{1}{2}\left(\phi+\chi-\phi^{\prime}-\chi^{\prime}\right) \\
\gamma=\frac{1}{2}\left(\phi-\chi+\phi^{\prime}-\chi^{\prime}\right), \sigma=\frac{1}{2}\left(\phi-\chi-\phi^{\prime}+\chi^{\prime}\right)
\end{array}\right\} .
$$

These equations define an orthogonal transformation of $\mathbb{R}^{4}$; (see [28]). By the arguments given in Section 3 we have therefore

$$
d \mu_{c}(\phi) d \mu_{c}(\chi) d \mu_{C}\left(\phi^{\prime}\right) d \mu_{c}\left(\chi^{\prime}\right)=d \mu_{c}(\alpha) d \mu_{c}(\beta) d \mu_{c}(\gamma) d \mu_{c}(\sigma)
$$

The inverse of (A.2.2) is given by

$$
\left.\begin{array}{c}
\phi=\frac{1}{2}(\alpha+\beta+\gamma+\sigma), \chi=\frac{1}{2}(\alpha+\beta-\gamma-\sigma), \\
\phi^{\prime}=\frac{1}{2}(\alpha-\beta+\gamma-\sigma), \chi^{\prime}=\frac{1}{2}(\alpha-\beta-\gamma+\sigma)
\end{array}\right\} .
$$

Equations (A.2.4) and the trigonometric identities used in Section 3 give $\cos \phi+\cos \chi+\cos \phi^{\prime}+\cos \chi^{\prime}$

$$
=\left\{\cos \left(\frac{\alpha}{2}\right) \cos \left(\frac{\beta}{2}\right) \cos \left(\frac{\gamma}{2}\right) \cos \left(\frac{\sigma}{2}\right)+\sin \left(\frac{\alpha}{2}\right) \sin \left(\frac{\beta}{2}\right) \sin \left(\frac{\gamma}{2}\right) \sin \left(\frac{\sigma}{2}\right)\right\},
$$


as is verified on three lines.

We define

$$
\Gamma=4 \int_{X} d \rho(x) \cos \left(\frac{\alpha\left(l_{x}\right)}{2}\right) \cos \left(\frac{\beta\left(l_{x}\right)}{2}\right) \cos \left(\frac{\gamma\left(l_{x}\right)}{2}\right) \cos \left(\frac{\sigma\left(l_{x}\right)}{2}\right)
$$

and

$$
\Sigma=4 \int_{X} d \rho(x) \sin \left(\frac{\alpha\left(l_{x}\right)}{2}\right) \sin \left(\frac{\beta\left(l_{x}\right)}{2}\right) \sin \left(\frac{\gamma\left(l_{x}\right)}{2}\right) \sin \left(\frac{\sigma\left(l_{x}\right)}{2}\right) .
$$

From (A.2.1), (A.2.3) and (A.2.5)-(A.2.6) we conclude $d \mu(\phi) d \mu(\chi) d \mu\left(\phi^{\prime}\right) d \mu\left(\chi^{\prime}\right)$

$$
=\Xi(C, \rho)^{-4} e^{z \Gamma} e^{z \Sigma} d \mu_{C}(\alpha) d \mu_{C}(\beta) d \mu_{C}(\gamma) d \mu_{C}(\sigma) \text {. }
$$

Next, let $\left\{f_{j}\right\}$ be some sequence of vectors in the real Hilbert space $\mathscr{H}$ (see Section 3$)$, and let $h(i, j, l, m)$ be a function with the property that

$$
\sum_{i, j, l, m} h(i, j, l, m) c_{i} c_{j} c_{l} c_{m} \geq 0
$$

for all real sequences $\left\{c_{j}\right\}$. Furthermore, let $\langle-\rangle$ be the expectation given by the measure defined in (A.2.7). Expanding $e^{z \Gamma}$ and $e^{z \Sigma}$ in the measure defined by the r.h.s. of (A.2.7) we find

$$
\begin{aligned}
\sum_{i, j, l, m} h(i, j, l, m)\left\langle\alpha\left(f_{i}\right) \beta\left(f_{j}\right) \gamma\left(f_{l}\right) \sigma\left(f_{m}\right)\right\rangle \\
=\Xi(C, \rho)^{-4} \sum_{k, k^{\prime}=0}^{\infty} \frac{z^{k+k^{\prime}}}{k !\left(k^{\prime}\right) !} \int_{X^{k}} \prod_{i=1}^{k} d \rho\left(x_{i}\right) \int_{X^{k^{\prime}}} \prod_{j=1}^{k^{\prime}} d \rho\left(y_{j}\right) \\
\quad \cdot \sum_{i, j, l, m} h(i, j, l, m)\left\langle\alpha\left(f_{i}\right)\right\rangle\left((x)_{k}(y)_{k^{\prime}}\right) \\
\quad \cdot\left\langle\beta\left(f_{i}\right)\right\rangle\left((x)_{k}(y)_{k^{\prime}}\right)\left\langle\gamma\left(f_{l}\right)\right\rangle\left(\left(x_{k}\right)(y)_{k^{\prime}}\right)\left\langle\sigma\left(f_{m}\right)\right\rangle\left((x)_{k}(y)_{k^{\prime}}\right),
\end{aligned}
$$

where $\langle\alpha(f)\rangle\left((x)_{k}(y)_{k^{\prime}}\right)$

$$
=\int d \mu_{C}(\alpha) \alpha(f) \prod_{i=1}^{k} \cos \left(\frac{\alpha\left(l_{x_{i}}\right)}{2}\right) \prod_{j=1}^{k^{\prime}} \sin \left(\frac{\alpha\left(l_{y_{j}}\right)}{2}\right)=\langle\beta(f)\rangle\left((x)_{k}(y)_{k^{\prime}}\right)=\ldots
$$

By (A.2.8) and (A.2.10) the integrand of each term in the sum over $k$ and $k^{\prime}$ on the r.h.s of (A.2.9) is non-negative. Hence

$$
\sum_{i, j, l, m} h(i, j, l, m)\left\langle\alpha\left(f_{i}\right) \beta\left(f_{j}\right) \gamma\left(f_{l}\right) \sigma\left(f_{m}\right)\right\rangle \geq 0
$$

Using the fact that $\left\langle\longrightarrow\right.$ is even in $\phi, \chi, \phi^{\prime}$, and $\chi^{\prime}$ we verify easily that

$$
4\left\langle\alpha\left(f_{i}\right) \beta\left(f_{j}\right) \gamma\left(f_{l}\right) \sigma\left(f_{m}\right)\right\rangle=\left\langle\phi\left(f_{i}\right) \phi\left(f_{j}\right) \phi\left(f_{l}\right) \phi\left(f_{m}\right)\right\rangle_{C, \rho}^{C}
$$

by reexpressing $\alpha, \beta, \gamma$ and $\sigma$ in terms of $\phi, \chi, \phi^{\prime}, \chi^{\prime}$ by means of (A.2.2) and using the fact that $\phi, \chi, \phi^{\prime}$ and $\chi^{\prime}$ are identically distributed. Inequality (A.2.11) and (A.2.12) give the "inverse" Lebowitz inequality, so that Theorem 4.6 is proven.

We leave it to the reader to apply (A.2.2), (A.2.5) and (A.2.7) to the proof of many other inequalities of the type of the "inverse" Lebowitz inequality. 


\section{References}

1. Albeverio, S., Hфegh-Krohn, R. : Commun. math. Phys. 30, 171 (1973); Edwards, S., Lenard, A. : J. Math. Phys. 3, 778 (1962)

2. Brydges, D. : A rigorous approach to Debye screening in dilute classical Coulomb systems. Rockefeller preprint (1977)

3. Brydges, D., Federbush, P. : Commun. math. Phys. 49, 233 (1976); 53, 19 (1977)

4. Coleman, S. : Phys. Rev. D11, 2088 (1975)

5. Fortuin, C., Kasteleyn, P., Ginibre, J. : Commun. math. Phys. 22, 89 (1971)

6. Fröhlich, J. : Commun. math. Phys. 47, 233 (1976); Renormalization theory (eds. G. Velo, A. S. Wightman). Nato Adv. St. Inst. Series C. Dordrecht-Boston: Reidel 1976

7. Fröhlich, J. : Proceedings of the International Conference on Mathematical Physics, Rome (1977)

8. Fröhlich, J., Park, Y. M. : Helv. Phys. Acta 50, 315 (1977)

9. Fröhlich, J., Park, Y. M. : In preparation

10. Fröhlich, J., Seiler, E. : Helv. Phys. Acta 49, 889 (1976)

11. Fröhlich, J., Simon, B. : Ann. Math. 105, 493 (1977)

12. Ginibre, J.: Some applications of functional integration in statistical mechanics. In: Statistical mechanics and quantum field theory, Les Houches 1970 (eds. C. DeWitt, R. Stora). New York: Gordon and Breach 1971 (See also Refs. to original articles given there)

13. Ginibre, J. : Commun. math. Phys. 16, 310 (1970)

14. Guerra, F., Rosen, L., Simon, B. : Ann. Math. 101, 111 (1975)

15. Israel, R. : Princeton series in physics, Princeton: University Press (to appear), (based on this author's PhD thesis, 1975)

16. José, J. V., Kadanoff, L. P., Kirkpatrick, S., Nelson, D. R. : IBM Res. Pep. RC 6428 (27401) (1977) and Refs. given therein

17. Lebowitz, J. L. : Commun. math. Phys. 28, 313 (1972)

18. Lebowitz, J. L. : Commun. math. Phys. 35, 87 (1974)

19. Lebowitz, J. L., Martin-Löf, A. : Commun. math. Phys. 25, 276 (1972); Lebowitz, J. L. : Proceedings of the International Conference on Mathematical Physics, Rome (1977) (see Ref. [7])

20. Lebowitz, J. L., Presutti, E. : Commun. math. Phys. 50, 195 (1976)

21. Lieb, E. H., Lewbowitz, J. L. : Advanc. Math. 9, 316 (1972); Lieb, E. H. : Rev. Mod. Phys. 48, 553 (1976)

22. Nelson, E. : J. Math. Phys. 5, 332 (1964)

23. Nelson, E. : Constructive quantum field theory (eds. G. Velo, A. S. Wightman). Lecture notes in physics, Vol. 25. Berlin-Heidelberg-New York: Springer 1973

24. Park, Y. M. : J. Math. Phys. (to appear) (1977)

25. Ruelle, D. : Statistical mechanics. Reading-London-Amsterdam-Tokyo: W. A. Benjamin 1969

26. Ruelle, D. : J. Math. Phys. 12, 901 (1971); Helv. Phys. Acta 45, 215 (1972); Fröhlich, J. : Helv. Phys. Acta 48, 355 (1975)

27. Simon, B. : Commun. math. Phys. 31, 127 (1973)

28. Sylvester, G. : MIT PhD thesis (1976); J. Stat. Phys. (to appear) (1977)

29. Siegert, A. J. F. : Physica 26, 30 (1960)

Communicated by E. Lieb

Received November 15, 1977 